\title{
From hydrodynamic lubrication to many-body interactions in dense suspensions of active swimmers
}

\author{
Natsuhiko Yoshinaga ${ }^{1,2,3}$ and Tanniemola B. Liverpool ${ }^{3,4,5}$ \\ 1 WPI - Advanced Institute for Materials Research, Tohoku University, Sendai 980-8577, Japan, e-mail: \\ yoshinaga@tohoku.ac.jp \\ 2 MathAM-OIL, AIST, Sendai 980-8577, Japan \\ 3 The Kavli Institute for Theoretical Physics, University of California, Santa Barbara, CA 93106, USA \\ 4 School of Mathematics, University of Bristol, Bristol, BS8 1TW, UK, e-mail: t.liverpool@bristol.ac.uk \\ ${ }^{5}$ BrisSynBio, Life Sciences Building, Tyndall Avenue, Bristol, BS8 1TQ, UK
}

Received: date / Revised version: date

\begin{abstract}
We study how hydrodynamic interactions affect the collective behaviour of active particles suspended in a fluid at high concentrations, with particular attention to lubrication forces which appear when the particles are very close to one another. We compute exactly the limiting behaviour of the hydrodynamic interactions between two spherical (circular) active swimmers in very close proximity to one another in the general setting in both three and (two) dimensions. Combining this with far-field interactions, we develop a novel numerical scheme which allows us to study the collective behaviour of large numbers of active particles with accurate hydrodynamic interactions when close to one another. We study active swimmers whose intrinsic flow fields are characterised by force dipoles and quadrupoles. Using this scheme, we are able to show that lubrication forces when the particles are very close to each other can play as important a role as long-range hydrodynamic interactions in determining their many-body behaviour. We find that when the swimmer force dipole is large, finite clusters and open gel-like clusters appear rather than complete phase separation. This suppression is due to near-field lubrication interactions. For swimmers with small force dipoles, we find surprisingly that a globally polar ordered phase appears because near field lubrication rather than long-range hydrodnamics dominate the alignment mechanism. Polar order is present for very large system sizes and is stable to fluctuations with a finite noise amplitude. We explain the emergence of polar order using a minimal model in which only the leading rotational effect of the near-field interaction is included. These phenomena are also reproduced in two dimensions.
\end{abstract}

PACS. 87.18.Hf Pattern formation in cellular populations - 64.75.Xc Phase separation and segregation in colloids - 47.63.Gd swimming of Microorganisms - 47.15.G- low-Reynolds number fluid flow

\section{Introduction}

Active materials are condensed matter systems which contain components that are self-driven out of equilibrium, and have been studied as inspiration for new smart materials and as a framework to understand aspects of cell motility [1,2,3]. They are characterised by a plethora of fascinating non-equilibrium collective phenomena such as swirling, alignment, pattern formation, dynamic cluster formation and phase separation $[4,5,6,7,8$, which have recently generated much interest. This phenomenology has inspired models of active suspensions involving assemblies of actively moving objects in far-from-equilibrium states at various length and time scales ranging from animals to cells and microorganisms. Theoretical descriptions of active systems range from continuum models [1,9] to discrete

Send offprint requests to: collections of self-propelled active particles [4]. A recent influential classification of self-propelled active particle systems has grouped them into dry and wet systems [1. Dry systems do not have momentum conserving dynamics. Examples include Vicsek models [4,9] and Active Brownian particle $(\mathrm{ABP})$ models interacting via soft repulsive potentials [10,11, 12, 13. Wet systems conserve momentum via a coupling to a fluid leading to hydrodynamic interactions between active particles. Microorganism suspensions are a typical example of this class. Inspired by these systems, several models have been proposed such as squirmers driven by surface deformations [14,15] and Janus particles driven by surface chemical reactions [16], which under certain conditions can be mapped to squirmers with tangential deformations [17. While dry models are attractive due to their computational tractability, it is not clear under which conditions they are useful models for the increasing number of experiments on micron sized 
self-propelled particles 7, 8. Hence it is essential to clarify the role of hydrodynamic interactions. In this work, we systematically construct equations of motion for wet active particles, namely, the dynamics of their position and orientation.

When hydrodynamic interactions are present, the motion of a particle is affected by long-range interactions from other particles due to both fluid flow and pressure. This results in many-body effects due to higher-order multipoles (see sect.2.4). In addition, even the two-body interaction between particles in close proximity (near-field) has non-trivial singular behaviour, requiring either exquisitely fine meshes between the gap or prior knowledge of analytical solutions in this region $[18,19,20,21,22,23,24$. It remains a significant challenge to overcome these technical hurdles and hence obtain an understanding of collective behaviour 25. Because numerical simulations with hydrodynamics require significantly more computational power (for example, $\mathcal{O}\left(N^{3}\right)$ for Stokesian Dynamics, and $\mathcal{O}\left((L / b)^{d}\right)$ for Lattice Boltzmann method, where $N, L, b$, and $d$ are the number of particles, the system size, the size of a mesh, and space dimension, respectively), studies of these systems to date contain relatively small number of particles compared with studies of ABPs and the Vicsek model [26, 27, 28,29]. It has been noted however that in those dry active systems, macroscopic behaviour, such as the spontaneous breaking of symmetries and the emergence of global order, are strongly affected by finite size effects. This leads one to worry if studies based on small numbers of particles can be accurately used to study macroscopic behaviour of wet active systems. As a result, the far-field approximation 30 has often been used to account for hydrodynamic interactions 31,32 . Within this approximation, swimmers are represented as point dipoles (denoted as $v_{2}$ in this work) and quadrupoles (denoted by $v_{1}$ ). This approximation while able to study large numbers of active particles only makes sense for describing very dilute suspensions because the far-field approximation is no longer valid when the swimmers are close to one another. So for non-dilute suspensions, there is a need for studies of wet active systems which take account of hydrodynamics accurately for small swimmer separations and can also deal with large number of particles.

We have recently proposed a model including both long-range interactions and accurate near-field lubrication forces at the level of two-body interactions, sacrificing however, accuracy at intermediate length scales. Using it we have studied and demonstrated a number of new classes of collective behaviours of squirmers in large systems 33. An outline of our method is schematically illustrated in fig. 1. We study active swimmers (squirmers) which when isolated generate an intrinsic flow in the farfield that are equivalent to those characterised by force dipoles and quadrupoles, the magnitudes of which, can be controlled as microscopic parameters. When two particles are far apart, the flow field is dominated by the far-field interaction, which is a flow field generated by an isolated swimmer with perturbation due to the other swimmer. When two particles approach each other, their transla- tional and rotational motion is dominated by the nearfield interaction. The near-field motion and rotation are obtained by decomposing the flow field into its passive (see figs. 1(B) and (D)) and active (see figs. 1(A) and (C)) parts and solving for the exact hydrodynamic flow fields in the lubrication limit. In this work, we will discuss the details of our new methodology and present more precise analyses of the results produced by the model. In addition, we present a detailed comparison between our method and other methods to handle hydrodynamic interactions in active materials. We point out a number of aspects of the problem that are sometimes overlooked. We also present a detailed analysis of the mechanisms of aggregation of the active swimmers. This involves introducing and characterising quantitatively, dynamic and static clusters, which appear when the absolute value of the dipole force is large $\left|v_{2}\right| \gg 0$. When $\left|v_{2}\right| \simeq 0$, a globally polar ordered phase appears as reported in 33 and [18, 34. Here, we confirm this result by showing polar order is robust to fluctuations of finite amplitude. Further more we are able to identify its origin and show that a global polar phase appears in a simplified model where only the rotational near-field interaction and steric interactions are included. Finally, our model is applied to two-dimensional squirmers, that is, particles move in the two-dimensional plane with purely two-dimensional hydrodynamic interactions. We also present general and explicit formulas for the farfield and near-field fluid flows, which, to our knowledge, have not been reported elsewhere before.

This paper is organised as follows: In sect. 2, we describe our model squirmers. The flow field generated by an isolated squirmer is obtained exactly. Then, the interactions between two squirmers are analytically calculated in two asymptotic limits; when the two swimmers (i) are far apart and (ii) are nearly touching. A comparison to previous studies is also presented. Readers who are interested only in the results of analytical calculations of the interactions may skip the beginning of the section and go directly to eqs. 28), (29), and the following discussions. We use the result of the interactions in sect. $2 \mathrm{~B}$ to perform numerical simulations of the collective behaviour of many squirmers in sect. 3. We also consider two-dimensional systems, which are discussed in sect. 3.4. In sect. 4, the dynamics of a collision of a pair of squirmers are analysed in detail from their initial approach to their eventual separation. We conclude with sect. 5, which summarizes our results. The technical details are outlined in the Appendices.

\section{Model}

Each particle (squirmer) is characterized by its position and orientation $\left(\mathbf{r}^{(i)}, \mathbf{p}^{(i)}\right)$ with dynamics given by

$$
\begin{aligned}
\dot{\mathbf{r}}^{(i)} & =\mathbf{u}^{(i)} \\
\dot{\mathbf{p}}^{(i)} & =\boldsymbol{\omega}^{(i)} \times \mathbf{p}^{(i)}
\end{aligned}
$$

The translational and angular velocities of each particle are denoted by $\mathbf{u}^{(i)}$ and $\boldsymbol{\omega}^{(i)}$, respectively. To obtain the 


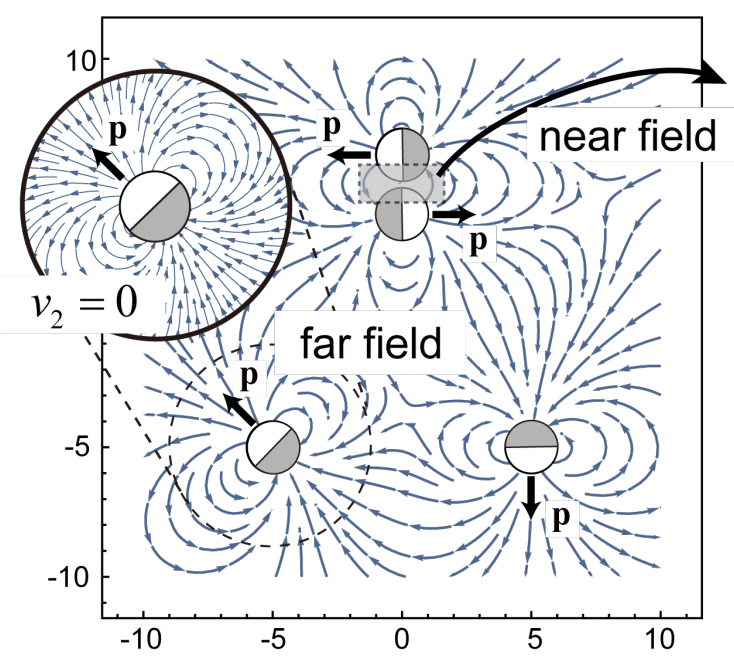

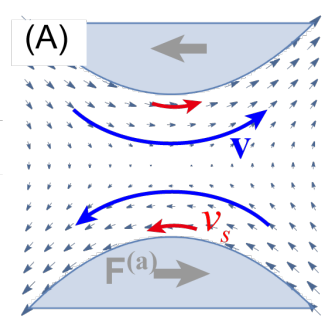

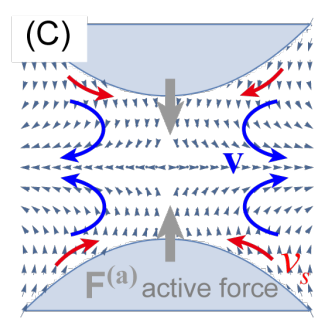

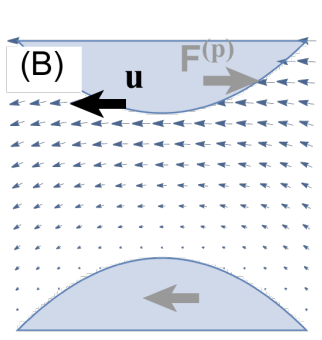

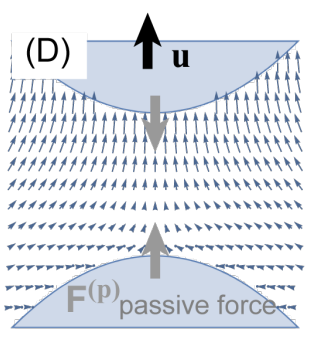

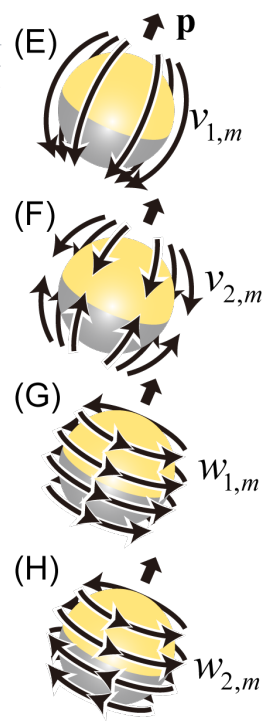

Fig. 1. (Colour Online) (Left) Schematic interaction between swimmers and surrounding flow. Each particle creates a leading order multipolar flow, which perturbs the translational and rotational motion of others in quite different ways in the far-field (when swimmers are well separated) and near-field (in close proximity to one another). (Right) The near-field flow field around shearing (A,B) and converging (C,D) swimmers. The active problems are shown in (A) and (C), and the passive problems are shown in (B) and (D). The direction of motion $\mathbf{u}$ and the surface slip flow are indicated by thick black and thin red arrows, respectively. The force $\mathbf{F}^{(a)}$ and $\mathbf{F}^{(p)}$ acting on each swimmer in those configurations is shown by a gray arrow for active and passive problems, respectively. As a guide to the eye, the blue arrows show fluid flow. (E-H) Schematic of the slip velocity on the surface of a swimmer and the flow it generates. The arrows show the tangential flow in the direction, $\boldsymbol{\Psi}_{l m}(\theta, \varphi)$ for $l=1$ (E) and $l=2(\mathrm{~F})$, and in the direction, $\boldsymbol{\Phi}_{l m}(\theta, \varphi)$ for $l=1(\mathrm{G})$ and $l=2(\mathrm{H})$ in eq. 6 .

velocities, we solve for the fluid mediated interaction between all the squirmers. The fluid is taken as incompressible in the vanishing Re limit :

$$
\begin{array}{r}
\eta \nabla^{2} \mathbf{v}-\nabla p=0 \\
\nabla \cdot \mathbf{v}=0
\end{array}
$$

where $\eta$ is viscosity, $\mathbf{v}(\mathbf{r})$ is the velocity, and $p(\mathbf{r})$ the pressure. The boundary condition on the swimmer surface is a sum of rigid translational, $\mathbf{u}$ and rotational, $\boldsymbol{\omega}$ motion and an active slip flow, $\mathbf{v}_{s}$ driving self-propulsion:

$$
\begin{aligned}
\left.\mathbf{v}\right|_{\mathbf{r}=\mathbf{R}} & =\mathbf{u}+\boldsymbol{\omega} \times \mathbf{R}+\mathbf{v}_{s} \\
\mathbf{v}_{s} & =\sum_{l \geq 1, m}\left[v_{l m} \boldsymbol{\Psi}_{l m}(\theta, \varphi)+w_{l m} \boldsymbol{\Phi}_{l m}(\theta, \varphi)\right]
\end{aligned}
$$

for a swimmer with centre at the origin. The fluid velocity vanishes at infinity, $\left.\mathbf{v}\right|_{r \rightarrow \infty}=0$, with $\theta$ the angle with the $z$-axis and $\varphi$ with the $x$-axis on the $x y$-plane. The slip velocity $\mathbf{v}_{s}$ can be very efficiently expanded in vector spherical harmonics, $\boldsymbol{\Psi}_{l m}$ and $\boldsymbol{\Phi}_{l m}$ which span the space of tangent vectors on the sphere [35] (as done in eq. (6)). The vector spherical harmonics $\mathbf{Y}_{l m}(\theta, \varphi), \boldsymbol{\Phi}_{l m}(\theta, \varphi)$ and $\boldsymbol{\Psi}_{l m}(\theta, \varphi)$ are defined by

$$
\begin{aligned}
& \mathbf{Y}_{l m}(\theta, \varphi)=\hat{\mathbf{r}} Y_{l}^{m}(\theta, \varphi) \\
& \mathbf{\Psi}_{l m}(\theta, \varphi)=r \nabla Y_{l}^{m}(\theta, \varphi) \\
& \boldsymbol{\Phi}_{l m}(\theta, \varphi)=\mathbf{r} \times \nabla Y_{l}^{m}(\theta, \varphi),
\end{aligned}
$$

where $Y_{l}^{m}(\theta, \phi)$ are the Laplace spherical harmonics. The spherical harmonics, $Y_{l}^{m}(\theta, \varphi)$ are defined here as

$$
Y_{l}^{m}(\theta, \varphi)=\mathcal{N}_{l m} P_{l}^{m}(\cos \theta) e^{i m \varphi}
$$

with $P_{l}^{m}(\cos \theta)$, the associated Legendre polynomial of degree $l$ and order $m$. We use the normalization convention $\mathcal{N}_{l m}=\sqrt{\frac{(2 l+1)(l-m) !}{4 \pi(l+m) !}}$

The second term in eq. (6) characterised by its coefficient $w_{l m}$ represents rotational slip associated with spinning motion (see fig. 1 $(\mathrm{G}-\mathrm{H})$ ). This does not, however, mean $w_{l m}=0$ ensures axissymetric flow around the particle even when it is isolated. We discuss this issue below eq. (12). For simplicity, in this work, we neglect this term in the following and from now on set $w_{l m}=0$. The swimmer axis

$$
\mathbf{p}=(\cos \alpha \sin \beta, \sin \alpha \sin \beta, \cos \beta)
$$

is a unit vector with azimuthal, $\alpha$ and polar, $\beta$ angles (see fig. 2(A) and its projection onto the $x z$ plane shown in fig. $2(\mathrm{~B}))$.

For uniaxial particles, $v_{l m}$ can, without loss of generality, be decomposed into a magnitude $v_{l}$ and the angles $(\alpha$, $\beta)$ as $v_{l m}=\mathcal{D}_{m 0}(\alpha, \beta) v_{l}$ with Wigner matrix $\mathcal{D}_{m m^{\prime}}(\alpha, \beta)$ rotating a $2 l+1$-dimensional vector of the $v_{l m}$ [36]. This is explicitly expressed as

$$
v_{l m}=\sqrt{\frac{4 \pi}{2 l+1}} Y_{l}^{m *}(\beta, \alpha) v_{l},
$$



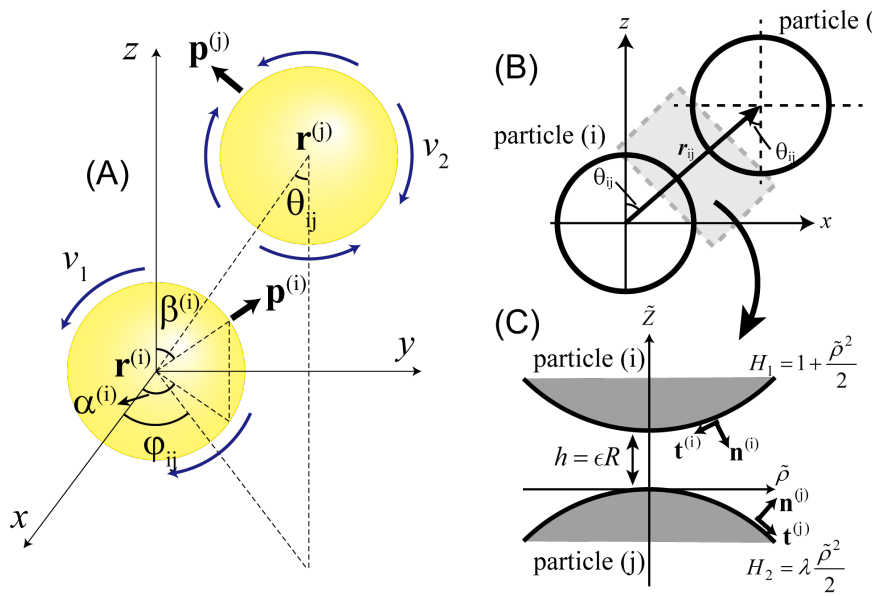

Fig. 2. (A) Characterising the state of the swimmer with position/orientation $\mathbf{r}, \mathbf{p}$ and its relation to the parameters $v_{1}, v_{2}, \alpha, \beta$ and the polar angles $\theta, \varphi$. (B) The coordinate for arbitrary position of the $i$ th and $j$ th particles. (C) The stretched coordinate used for calculation of the near-field interaction between the spheres (i) and (j) placed along the $z$-axis.

in terms of the modes $v_{l}$. Here, $Y_{l}^{m *}(\beta, \alpha)$ is the complex conjugate of $Y_{l}^{m}(\beta, \alpha)$. Note that in the double sum in eq. (6), for each $l ; m$ takes values $-l,-l+1, \ldots, 0, \ldots, l-$ $1, l$ and hence $v_{l m}$ can be represented by a $2 l+1$-dimensional vector. For $l=1$ and $l=2$, the vectors are shown in Appendix A. A uniaxial squirmer is characterised by the strength of the $l$-th mode, $v_{l}$, and its swimming orientation by the two angles $(\alpha$ and $\beta$ ). When the particle is not uniaxial, the $v_{l m}$ for $l \geq 2$ will have additional degrees of freedom.

The flow field and resulting motion for an isolated squirmer is summarized in the next section. The first, $l=1$ mode in eq. (6) is a quadrupolar flow with strength $v_{1}$ (i.e. it decays like $r^{-3}$ at large distances $r$ from the swimmer, detailed analysis shows that it is a potential flow source/sink dipole) 37 leading to translational motion while the second, $l=2$ mode is a dipole of strength $v_{2}$ (decays like $r^{-2}$ ) 37. In much of the literature on squirmers [14,15, the surface slip flow $\mathbf{v}_{s}$ in eq. (6) is expanded in terms of Legendre polynomials assuming an axisymmetric flow profile, with coefficients $B_{l}$ which can be obtained using the orthogonality of the Legendre polynomials. We can relate the parameters $B_{l}$ of these papers, to the coefficients $v_{l}$ of our expansion in terms of the vector spherical harmonics:

$$
B_{l}=-v_{l} \sqrt{\frac{(2 l+1)}{4 \pi}} \frac{l(l+1)}{2}
$$

leading to $v_{1}=-\sqrt{4 \pi / 3} B_{1}$ and $v_{2}=(-2 / 3) \sqrt{\pi / 5} B_{2}$, and thus the squirmer parameter $\beta_{2 / 1}\left(B_{2} / B_{1}=\beta\right.$ in the conventional notation $)$ is $\beta_{2 / 1}=(3 \sqrt{5 / 3}) v_{2} / v_{1} \simeq 3.87 v_{2} / v_{1}$

\subsection{Flow field around an isolated squirmer}

In this section, we give the explicit forms of the flow field generated by an isolated non-spinning squirmer in terms of the vector spherical harmonics. The velocity field and the pressure field are in general expressed as

$$
\begin{aligned}
& \mathbf{v}(\mathbf{r})=\sum_{l \geq 1, m}\left[f_{l m}^{(t)}(r) \boldsymbol{\Psi}_{l m}(\theta, \varphi)+f_{l m}^{(n)}(r) \mathbf{Y}_{l m}(\theta, \varphi)\right] \\
& p(\mathbf{r})=\sum_{l \geq 1, m} P_{l m}\left(\frac{R}{r}\right)^{l+1} Y_{l}^{m}(\theta, \varphi) .
\end{aligned}
$$

The coefficient functions $f_{l m}^{(t)}, f_{l m}^{(n)}$, and $P_{l m}$ are obtained by solving the Stokes equation (34) with the effective slip boundary conditions eq. 6 .

The coefficients for the first mode, $l=1$ are

$$
\begin{aligned}
f_{1, m}^{(n)} & =u_{m}\left(\frac{R}{r}\right)^{3} \\
f_{1, m}^{(t)} & =-\frac{1}{2} u_{m}\left(\frac{R}{r}\right)^{3} \\
P_{1, m} & =0 .
\end{aligned}
$$

For the higher modes, $l \geq 2$, they are

$$
\begin{aligned}
f_{l m}^{(n)} & =\frac{l(l+1)}{2} v_{l m}\left[\left(\frac{R}{r}\right)^{l}-\left(\frac{R}{r}\right)^{l+2}\right] \\
f_{l m}^{(t)} & =-\frac{v_{l m}}{2}\left[(l-2)\left(\frac{R}{r}\right)^{l}-l\left(\frac{R}{r}\right)^{l+2}\right] \\
P_{l m} & =\frac{\eta}{R} l(2 l-1) v_{l m} \\
u_{m} & =-\frac{2}{3} v_{1, m} .
\end{aligned}
$$

The self-propulsion velocities of the particle are expressed in Cartesian coordinates as

$$
\begin{aligned}
\mathbf{u} & =\sum_{m} u_{m}\left[\mathbf{Y}_{1, m}(\theta, \varphi)+\mathbf{\Psi}_{1, m}(\theta, \varphi)\right] \\
& =\left(\begin{array}{c}
\mathcal{N}_{1,1}\left(-u_{1}+u_{-1}\right) \\
i \mathcal{N}_{1,1}\left(-u_{1}-u_{-1}\right) \\
\mathcal{N}_{1,0} u_{0}
\end{array}\right) . \\
\boldsymbol{\omega} & =0 .
\end{aligned}
$$

It is noteworthy that (1) the isolated squirmer is force and torque free by construction due to the absence of the $1 / r$ tern in the $l=1$ modes and (2) the self-propulsion speed depends only on the first, $l=1$ mode and is independent of the higher modes, $l \geq 2$. Since we do not consider selfrotating squirmers, the angular velocity of the isolated swimmer is identically zero.

We use the vector spherical harmonics for expansion of an active slip flow and a surrounding flow. This is because two tangential flows associating with translation and selfrotation become clearer (See eq. (6)). Nevertheless, it is possible to express these flow in terms of Cartesian tensors. In Appendix B, we demonstrate an explicit translation of a fluid flow around an isolated swimmer into the form of Cartesian tensors. 


\subsection{Pairwise interactions between squirmers}

When two squirmers are present, the flow field generated by one will affect the other and hence lead to modification of the self-propulsion velocities. To calculate the modified flow and hence the effective pairwise hydrodynamic interactions between two squirmers, we solve the Stokes equation (34) with slip boundary conditions, eqs. (6) on the surface of both swimmers taking into account both of their orientations (denoted by the unit vector $\mathbf{p}^{(i)}$ ) which are chosen arbitrarily. This gives rise to modified velocities, $\mathbf{u}^{(i)}, \boldsymbol{\omega}^{(i)}$ for each squirmer which will also depend on the position and orientation of the other. To compute the velocities, $\mathbf{u}^{(i)}, \boldsymbol{\omega}^{(i)}$ we split the problem into two parts, a force and torque acting on the sphere with: 1st, slip boundary conditions without translational and rotational motion, and 2nd with the non-slip boundary conditions undergoing rigid-body motion $\mathbf{u}^{(i)}$ and $\boldsymbol{\omega}^{(i)}$. We shall call the former the active force (torque), $\mathbf{F}^{(a)}\left(\mathbf{T}^{(a)}\right)$ and the latter the passive force (torque), $\mathbf{F}^{(p)}\left(\mathbf{T}^{(p)}\right)$. The force and torque-free conditions imply,

$$
\begin{aligned}
\mathbf{F}^{(a)}+\mathbf{F}^{(p)} & =0 \\
\mathbf{T}^{(a)}+\mathbf{T}^{(p)} & =0 .
\end{aligned}
$$

The passive force and torque are proportional to $\mathbf{u}^{(i)}$ and $\boldsymbol{\omega}^{(i)}$ while the active force and torque are proportional to $v_{l m}$. Therefore the force and torque-free conditions are exactly expressed by

$$
\mathbf{L}^{(i, j)} \cdot\left(\begin{array}{c}
\mathbf{u}^{(j)} \\
\boldsymbol{\omega}^{(j)}
\end{array}\right)=\mathcal{L}_{l m}^{(i, j)} \cdot v_{l m}^{(j)}
$$

where the superscript $(i)$ denotes $i$ th particle. $\mathbf{L}$ is called the passive resistance matrix, likewise $\mathcal{L}$, the active resistance matrix, The resistance matrices depend only on the shapes of the particles and their relative positions $\left(\mathbf{r}_{i j}\right.$ being the vector between their centers, see fig. 2(A)). The matrices $\mathbf{L}, \mathcal{L}$ can be calculated exactly for pairs of particles in two asymptotic limits : (1) when their separation, $h_{i j}=r_{i j}-2 R, r_{i j}=\left|\mathbf{r}_{i j}\right|$ is much less than their radius (near-field, fig. 2(C)) and (2) when their separation is much greater than their radius (far-field, fig. 2(B)). There is long history of calculation of the passive matrix [38, 39. Here we compute the active resistance matrix for both farfield and near-field in the general setting. Previous near field results have been obtained only for axisymmetric surface flow-fields. We note that non-axisymmetric flow fields around isolated swimmers, however have been considered before, e.g. in 40.

It should be noted that to obtain the velocity and angular velocity for swimmers oriented in arbitrary directions, one must compute eqs. (25) and (26) for all possible orientation directions. Hence, below, we decompose the problem into the eight possible motions (see fig. 3) and calculate the velocities for two arbitrarily oriented swimmers in eqs. (28) and (29). This has not been achieved before; we will compare our analytical results with previous studies in sect. 2.4.
The equations (1), 2), and 27) form a closed complete dynamical system. We use a general form for the velocities valid in both far and near field limits:

$$
\begin{aligned}
& \mathbf{u}^{(i)}=\mathbf{u}_{0}^{(i)} \lambda^{(i)}+\sum_{j \neq i, l, m}\left[u_{l m, \|}^{(j)} \mathbf{Y}_{l m}^{(j i)}+u_{l m, \perp}^{(j)} \mathbf{\Psi}_{l m}^{(j i)}\right] \\
& \boldsymbol{\omega}^{(i)}=\sum_{j \neq i, l, m} \omega_{l m}^{(j)} \boldsymbol{\Phi}_{l m}^{(j i)} .
\end{aligned}
$$

The isolated squirmer moves with the velocity

$$
\begin{aligned}
\mathbf{u}_{0}^{(i)} & =u_{0} \mathbf{p}^{(i)} \\
u_{0} & =-\frac{2}{3} \sqrt{\frac{3}{4 \pi}} v_{1} .
\end{aligned}
$$

$\lambda^{(i)}=1$ when the $i$ th particle is away from near-field region of any other particles and $\lambda^{(i)}=0$ otherwise. For a pair of squirmers (labelled $i, j$ ) with arbitrary positions (and orientation), we can define a set of spherical coordinates with relative positions $\mathbf{r}_{i j}$ and relative angles $\theta_{i j}$ and $\varphi_{i j}$ (see fig. 22. We denote $\mathbf{Y}_{l m}\left(\theta_{j i}, \varphi_{j i}\right)=\mathbf{Y}_{l m}^{(j i)}$ and $\theta_{j i}=\pi-\theta_{i j}$ and $\varphi_{j i}=\pi+\varphi_{i j} . \theta_{i j}$ is the polar angle of the vector between the centers of the $i$ th and $j$ th particles and $\varphi_{i j}$ is its azimuthal angle.

The symmetry of this interaction is obtained from the following property of the spherical harmonics:

$$
Y_{l}^{m}\left(\theta_{j i}, \varphi_{j i}\right)=(-1)^{l} Y_{l}^{m}\left(\theta_{i j}, \varphi_{i j}\right) .
$$

Similarly, the vector spherical harmonics transform as

$$
\begin{gathered}
\mathbf{Y}_{l m}\left(\theta_{j i}, \varphi_{j i}\right)=(-1)^{l+1} \mathbf{Y}_{l m}\left(\theta_{i j}, \varphi_{i j}\right) \\
\boldsymbol{\Psi}_{l m}\left(\theta_{j i}, \varphi_{j i}\right)=(-1)^{l+1} \boldsymbol{\Psi}_{l m}\left(\theta_{i j}, \varphi_{i j}\right) \\
\boldsymbol{\Phi}_{l m}\left(\theta_{j i}, \varphi_{j i}\right)=(-1)^{l} \boldsymbol{\Phi}_{l m}\left(\theta_{i j}, \varphi_{i j}\right) .
\end{gathered}
$$

\subsubsection{Near-field}

The calculation of the near-field interaction is a conceptually straightforward, if technically complicated computation. For completeness, we briefly outline the main steps in this section. Without loss of generality, two spherical squirmers are placed along the $z$-axis (fig. 3). Then flow induced by the interaction is decomposed into parallel and perpendicular directions corresponding to the $\mathbf{Y}_{l m}$ and $\boldsymbol{\Psi}_{l m}$ terms. Since we may arbitrarily choose the $x$ and $y$ axes, we have two translational velocities $u_{x}$ and $u_{z}$ and two angular velocities $\omega_{y}$ and $\omega_{z}$ for each particle. These eight unknown velocities are determined by the eight configurations shown in fig. 3. In the suite, we neglect (d) and (h) in fig. 3 since we do not consider spinning motion.

When the two squirmers almost touch each other, we may assume the gap between two sphere surfaces $h$ is much smaller than their radius (see fig. $2(\mathrm{C})$ ). We introduce the small parameter

$$
\epsilon=\frac{h}{R}
$$



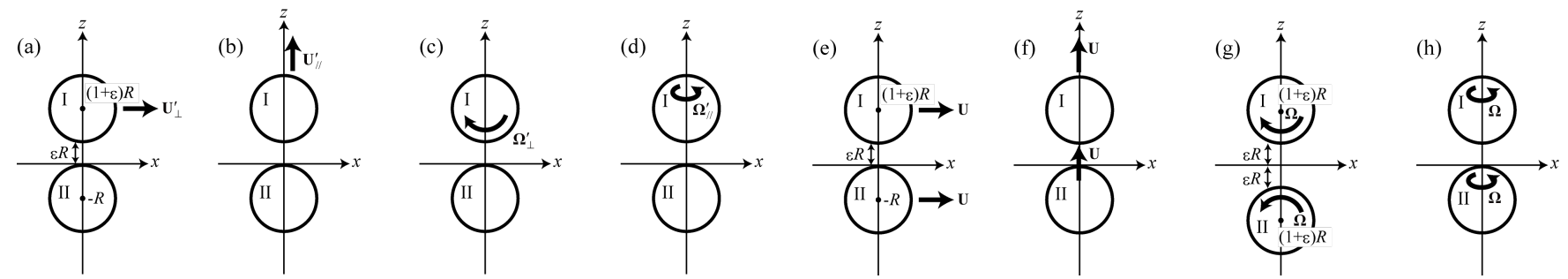

Fig. 3. The eight configurations for translational and rotational motion of two particles. The thick arrows show the direction of motion.

The asymptotic behaviour in the limit of small $\epsilon$ for the dynamics of rigid spheres moving slowly past each other (which we call the passive problem) has been studied by a number of authors [41,42, 43, 44, 45, 46, 47. Here we adapt and extend these results to the problem of squirmers in close proximity (the active problem). The leading order contribution in $\epsilon$ to the force and torque depends on the particular problem (configuration) considered (see fig. 3). It is well known that for the passive problem with the configuration (a)-(c) in fig. 3, the force and torque diverges with decreasing separation between the spheres, $\epsilon ; F^{(p)} \sim$ $1 / \epsilon$ for (b) 46 and $F^{(p)} \sim \log \epsilon$ for (a) and (c) 43, 47. For (e) and (f), both force and torque do not diverge as $\epsilon \rightarrow 0$ and thus $F^{(p)} \sim \mathcal{O}\left(\epsilon^{0}\right)$ and $T^{(p)} \sim \mathcal{O}\left(\epsilon^{0}\right)$ 42, 45. For $(\mathrm{g})$, the force is regular $F^{(p)} \sim \mathcal{O}\left(\epsilon^{0}\right)$ while the torque is singular $T^{(p)} \sim \mathcal{O}(\log \epsilon)$ 42. Although we have not considered spinning motion in this article - problems $(\mathrm{d})$ and (h) in fig. 3 , both the problems are not singular and thus $T^{(p)} \sim \mathcal{O}\left(\epsilon^{0}\right)$ for $(\mathrm{d})$ 47, 44 and $(\mathrm{h})$ 41.

When $\epsilon \rightarrow 0$, it is convenient to use the following stretched coordinate $(X, Y, Z)$ (see fig. $2(\mathrm{C})$ )

$$
\begin{aligned}
& x=\sqrt{\epsilon} X \\
& y=\sqrt{\epsilon} Y \\
& z=\epsilon Z .
\end{aligned}
$$

We scale lengths with $R$ defining, dimensionless coordinates $\tilde{X}=X / R, \tilde{Y}=Y / R$, and $\tilde{Z}=Z / R$. It is convenient to use cylindrical coordinates $(\rho, \varphi, z)$, where $\rho=x^{2}+y^{2}$, or equivalently stretched cylindrical coordinates $(\tilde{\rho}, \varphi, \tilde{Z})$ with $\tilde{\rho}=\sqrt{\tilde{X}^{2}+\tilde{Y}^{2}}$. We can without loss of generality place the centres of the two spheres (identified by labels $1,2)$ at (see fig. $2(\mathrm{~B}))$

$$
\begin{aligned}
& \mathbf{r}_{G}^{(1)}=(0,0,(1+\epsilon) R) \\
& \mathbf{r}_{G}^{(2)}=(0,0,-R) .
\end{aligned}
$$

The surfaces of the spheres are given by the boundary surfaces $H_{1}$ and $H_{2}$ respectively. The position at the boundary $\tilde{Z}=H_{1}$ and $\tilde{Z}=H_{2}$ is

$$
\begin{aligned}
& H_{1}=1+\frac{1}{2} \tilde{\rho}^{2}+\mathcal{O}(\epsilon)=H_{0}+\mathcal{O}(\epsilon) \\
& H_{2}=-\frac{1}{2} \tilde{\rho}^{2}+\mathcal{O}(\epsilon) .
\end{aligned}
$$

The squirmer slip velocity boundary condition, $\mathbf{v}_{\mathbf{s}}(\theta, \varphi)$ given in eq. (6) can be expanded in the coordinate system above. In the near field, the boundary condition is expanded as a series in $\epsilon$. The details of the calculation of the passive and active forces of the configuration (b) in fig. 3 are outlined in Appendix $\mathrm{C}$ and (a), (c), (g) in Appendix D.

The passive force and torque are linear functions of translational and rotational velocity, while the active force and torque are expressed in terms of the surface slip velocity. Using the generalized vector $\mathbf{U}=\left(\mathbf{u}^{(1)}, \mathbf{u}^{(2)}, \boldsymbol{\omega}^{(1)}, \boldsymbol{\omega}^{(2)}\right)$ and $\mathbf{V}_{s, l m}=\left(v_{l m}^{(1)}, v_{l m}^{(2)}\right)$, the problem is rewritten as

$$
\mathbf{L} \cdot \mathbf{U}=-\sum_{l, m} \mathcal{L}_{l m} \cdot \mathbf{V}_{s, m}
$$

where $\mathbf{L}$ is an invertible $4 d \times 4 d$ matrix while $\mathcal{L}_{l m}$ is $4 d \times$ $2(2 l+1)$ matrix. The passive and active resistance matrices $\mathbf{L}$ and $\mathcal{L}_{l m}$ are obtained from the analyses of the previous sections. The passive resistance matrix must be inverted to compute the velocities and angular velocities of the swimmers. Following [48, we include the diagonal terms corresponding to the force and torque of an translating and rotating isolated particle.

In the near field limit, our lubrication analysis enables us to obtain all the singular contributions as $\epsilon$ approaches zero. They arise from relative motion between the particles that give either shear or converging flow as shown in figs. 1(A)-(D).A similar analysis can be performed for motion perpendicular to the line between two centers. This corresponds to the problems (a), (c), (e), and (g).

When the separation between two sphere surfaces, $h_{i j}=$ $\epsilon R, \epsilon \ll 1$, is small compared to their size (see fig. 2 (B) and fig. 1), we systematically extend the passive lubrication calculation for nearby spheres with non-slip boundary conditions [49,50] to account for active slip boundary conditions of squirmers and hence obtain coefficients in eqs. 28) and (29) as

$$
\begin{aligned}
u_{l m, \|}^{N} & =-\epsilon \log \epsilon V_{l m}+\frac{1}{3} W_{1, m} \delta_{l, 1} \\
u_{l m, \perp}^{N} & =\frac{1}{2} V_{l m}+\frac{1}{3} W_{1, m} \delta_{l, 1} \\
\omega_{l m}^{N} & =-\frac{2}{5 R} W_{l m}
\end{aligned}
$$


with

$$
\begin{gathered}
V_{l m}=\frac{l(l+1)}{2}\left((-1)^{l} v_{l m}^{(i)}+v_{l m}^{(j)}\right) \\
W_{l m}=\frac{l(l+1)}{2}\left((-1)^{l} v_{l m}^{(i)}-v_{l m}^{(j)}\right) .
\end{gathered}
$$

The leading order velocity parallel to the center line is $\mathcal{O}(\epsilon \log \epsilon)$. This is due to the balance of the passive lubrication force $F^{(p)} \sim u / \epsilon$ and the active force $F^{(a)} \sim v_{l m} \log \epsilon$, which is singular but only logarithmic since the contribution from incompressibility is small here. This result is consistent with [18,51. Perpendicular motion and rotation are $\mathcal{O}(1)$ because both passive and active forces are logarithmic. Combining all of these, we obtain the result in eqs. (44)- 46 above.

\subsubsection{Far field}

The far-field interaction between the $i$ th and $j$ th particles follows from Faxen's laws [39] giving coefficients in eqs. 28 and (29) as

$$
\begin{aligned}
u_{1, m, \|}^{F} & =-\frac{2}{3} \frac{1}{\tilde{r}_{i j}^{3}} v_{1, m}, \\
u_{2, m, \|}^{F} & =\frac{3}{\tilde{r}_{i j}^{2}} v_{2, m}, \\
u_{1, m, \perp}^{F} & =\frac{1}{3} \frac{1}{\tilde{r}_{i j}^{3}} v_{1, m}, \\
\omega_{2, m}^{F} & =-\frac{3}{\tilde{r}_{i j}^{3}} v_{2, m} / R,
\end{aligned}
$$

and coefficients $l \geq 3$ vanish. Here $\tilde{r}_{i j}=r_{i j} / R$. Notice that in this limit the first, $l=1$ mode cannot induce rotation since it generates a potential flow. Hence for spheres, the first mode contributes to rotation only via multi-scattering effects.

\subsubsection{Implementation}

To describe the active resistance matrix for arbitrary separations between particles, we interpolate between two limiting expressions, the far-field and near field, using a tanh function centred at $r=2.5 R$ with width $0.1 R$, that is much smaller than the size of a particle. Note that eq. (44) gives rise to an effective repulsive interaction between two particles because the speed of convergence goes to zero as the separation between the particle surfaces become small. This is also true for passive particles, but the speed goes to zero slower for active particles due to the factor of $\log \epsilon$ in eq. (44). However due to fact that finite time steps are required for the numerical implementation of any multi-particle simulation, we require a short range repulsive interaction between all the particles to avoid overlap between them (i.e. to stop the separation between the centres becoming less than double the radius). In Stokesian Dynamics simulations, a short-range repulsive force, $\mathbf{F}_{i j}$ between swimmers $i$ and $j$, whose centres are separated by the vector $\mathbf{r}_{i j}$, is added to the equation of motion eq. (1) [52] (see also [53]), where

$$
\mathbf{F}_{i j}=F_{0} \frac{e^{-\epsilon / r_{s}}}{r_{s}\left(1-e^{-\epsilon / r_{s}}\right)} \hat{\mathbf{r}}_{i j}
$$

where $\epsilon=r_{i j} / R-2$ and $r_{s}=1 / 227$ [52]. A similar repulsive interaction has also been included in simulations of squirmers using the Boundary Element Method [18, 30. In this work, we experimented with a variety of repulsive interactions (including the one described above) and found that while a particular choice changes the positions of boundaries of different classes of behaviour, they do not lead to qualitative changes of the behaviour observed. Hence to obtain the phase diagrams which are the main result of this paper we used the common and wellknown truncated Lennard-Jones repulsive interaction, i.e. we we add a short-range repulsive force $\mathbf{F}_{i j}=-\partial U / \partial \mathbf{r}_{\mathbf{i j}}$ to eq. (1) where

$$
U(r)= \begin{cases}-2\left(\frac{2 R}{r_{i j}}\right)^{6}+\left(\frac{2 R}{r_{i j}}\right)^{12} & \text { for } r_{i j}<2 R \\ 0 & \text { otherwise }\end{cases}
$$

With this method, the particles overlap at most $2 \%$ of their radius for neutral swimmers at the maximum density that we used.

\subsection{Two-dimensional squirmers}

In two dimensions, the expansion of the slip velocity in terms of spherical harmonics is replaced by one in terms of sines and cosines

$$
v_{s}=\sum_{m=1}^{\infty}\left[v_{s, m} \sin m \theta+\tilde{v}_{s, m} \cos m \theta\right] .
$$

The radial and tangential direction are expressed in polar coordinates as $\mathbf{r}=(\cos \theta, \sin \theta)$ and $\mathbf{t}=(-\sin \theta, \cos \theta)$ (see fig. 2(B)). Then, the flow field of an isolated squirmer is expressed in terms of $\mathbf{r}_{m}=(\cos m \theta, \sin m \theta)$ and $\mathbf{t}_{m}=$ $(-\sin m \theta, \cos m \theta)$ (see also Appendix E for the explicit form of the flow field). The velocity of the particle is

$$
\mathbf{u}_{0}=-\frac{1}{2 \pi R} \int v_{s} \mathbf{t} d S=\frac{1}{2}\left(v_{s, 1} \mathbf{e}_{x}-\tilde{v}_{s, 1} \mathbf{e}_{y}\right) .
$$

In three dimensions, the dynamics is governed by eqs. 28 and 290 supplemented with eqs. (49)-(52) for far-field and eqs. (44)-46) for near-field. In two dimensions, the dynamics is governed by the following equations:

$$
\begin{aligned}
& \mathbf{u}^{(i)}=\mathbf{u}_{0}^{(i)} \lambda^{(i)}+\sum_{j \neq i, m}\left[u_{m, \|}^{(j)} \hat{\mathbf{r}}_{j i}+u_{m, \perp}^{(j)} \hat{\mathbf{t}}_{j i}\right] \\
& \omega^{(i)}=\sum_{j \neq i, m} \omega_{m}^{(j)}
\end{aligned}
$$


where expressions for $u_{m, \|}, u_{m, \perp}$ in the near-field and farfield regimes are given in Appendix $\mathrm{E}$ and

$$
\begin{aligned}
\hat{\mathbf{r}}_{i j, m} & =\left(\cos m \theta_{i j}, \sin m \theta_{i j}\right) \\
\hat{\mathbf{t}}_{i j, m} & =\left(-\sin m \theta_{i j}, \cos m \theta_{i j}\right) .
\end{aligned}
$$

In order to compute the interaction, we consider six problems in (a)-(c) and (e)-(g) in fig. 3. The explicit expressions are given in Appendix E. In contrast with threedimensional systems, the leading order velocity parallel to the center line is $\mathcal{O}(\sqrt{\epsilon})$. Perpendicular motion and rotation are the same as three-dimensional squirmers and proportional to $\mathcal{O}(1)$.

\subsection{Comparison to other work}

In this section, we compare the methods developed in this paper and previous work. First, we summarise the existing approaches. There are two methods commonly used to compute near-field interactions between spherical objects: bispherical (bipolar in two dimensions) coordinates and matched asymptotic analyses. Pair-wise interactions computed using bispherical coordinates are exact but the solution is expressed in terms of an infinite algebraic system of equations. The sum may be evaluated numerically with truncation after a finite number of terms, with more and more terms necessary as the two spheres approach each other 54. For large separations between spheres, this truncation is justified and the far-field interaction is reproduced.

For the near-field interaction, there is no general method to express this infinite series as an expansion in terms of the small separation $\epsilon$. We are only aware of one case in which this has been done, for perfectly aligned converging spheres (problem (b) in fig. 33) in which the series for solid spheres is approximated for small $\epsilon$ [55] (also used in [24] for a squirmer problem). The matched asymptotic analysis (used in this work) is an expansion of the solution for small $\epsilon$. Its results are, of course, not exact for arbitrary separation but become asymptotically exact when $\epsilon \ll 1$. In this particular case, the two approaches are identical both in the scaling with $\epsilon$ and the prefactor of the leading asymptotic term for $\epsilon \ll 1$ [55, 56].

The matched asymptotic method is appealing because it removes the need for cumbersome calculations of very many terms of the series obtained using the bispherical coordinate method. The analysis here, is based on the expansion of inner and outer regions. The inner region is obtained using the stretched coordinate system shown in fig. 2 and the outer region is obtained using the tangentsphere coordinate system [55,56]. The solution of each coordinate is matched at an appropriate limit. Clearly obtaining the full expansion will be as cumbersome as using bispherical coordinates. The point is that the leading order terms in the asymptotic expansion are a very good approximation to the full solution when the spheres are very close to each other. Calculating the leading order terms is relatively straightforward 49 and hence this gives a very efficient and accurate calculation of the interactions for spheres in close proximity. In this paper, we treat only the leading-order singularities which diverge as $\epsilon \rightarrow 0$, while for the finite $\mathcal{O}(1)$ terms, we use the values valid for isolated swimmers. This is because it is known that the $\mathcal{O}(1)$ terms obtained by matched asymptotics are not particularly accurate and typically these terms have been obtained either using numerical estimates of the exact expression using bispherical coordinates or from direct numerical solutions of the Stokes equation 38, which give values comparable to those of isolated swimmers.

In 24, an exact solution of the Stokes equation was computed for a axisymmetric arrangement of two squirmers using bispherical coordinates and the reciprocal theorem. However this work is of limited use for the study of collective behaviour (the subject of this paper) because it by construction addresses only a highly reduced set of interaction scenarios, those with axisymmetric flow fields. To study the collective behaviour one must be able to describe all possible directions of approach and orientations of the two swimmers. Since the system was assumed to be axisymmetric, only the motion and rotation corresponding to (b), (d), (f), and (h) in fig. 3 can be considered. In order to obtain the equation of motion of each swimmer, we need the functional form of the interaction for all possible directions. In the current work, we do not consider a spinning motion corresponding to $w_{l m} \neq 0$ in eq. (6), and therefore do not study rotational motion - problems (d) and (h) in fig. 3. Only the translational motion studied in problems (b) and (f) in fig. 3 can be addressed within the framework of 24. However, as there is no explicit formula discussed in this paper, a direct comparison is not possible.

Bispherical coordinates were also used in 23] to compute the interaction between two spherical Janus particles. In [23, both hydrodynamics (the Stokes equation) and a concentration field (the Laplace equation) are solved. In contrast to 24], axisymmetric motion and rotation were not assumed. Nevertheless, as the results of infinite series of coefficients were evaluated numerically a direct comparison is also not possible here.

We treat the near-field interactions in a similar way to [18,20, however while they compute passive and active resistance matrices using numerical tables, we obtain explicit formulas for the translational and angular velocity as eqs. (28) and (29). This allows us to perform simulations of this problem in an analogous way to active Brownian Particle simulations. We note also that while the force in the direction perpendicular to the centreline and torque were given in [18, an explicit expression of the force in the parallel direction was not provided in that paper. In [18, the active force in the perpendicular direction to the centreline between two spheres was obtained as $F_{x, 1}^{(a)}=-\pi \eta R d u_{s} \log \epsilon$, where $d u_{s}$ is "the difference between the squirming velocities of the two squirmers" and is expressed in our notation as $d u_{s}=\sum_{l, m= \pm 1} m \mathcal{N}_{l|m|} V_{l m}$. This result is exactly same as our result here, given by the first term in eq. 125) once we make the assumption of uniaxial slip velocity using eq. (12). Similarly, the torque 
was obtained as $T_{y, 1}^{(a)}=-\pi \eta R d u_{s} \log \epsilon$ when the sphere $1(2)$ is moving in the $x(-x)$ direction under the configuration shown in fig. 3 (a). This implies $W_{l m}=0$ in our notation, and then in the uniaxial case, eq. (127) gives exactly the same result using the properties of the spherical harmonics and the associated Legendre polynomials. We note that the analytical calculation in 18 is based on calculations of the force and torque when one sphere has a slip velocity and the other sphere is inert. Explicit formulas are given in 18 only for the force and torque acting on the squirming sphere, and not for inert one. Here we find that in order to evaluate the general form of the torque acting on both spheres when they are both squirming, the torque acting on the inert sphere is necessary because $(\mathrm{g})$ in fig. 3 has the same singularity as (c). It is not clear how this issue was treated in [18. We note also that although the we obtain identical expressions for the perpendicular forces and torques to 18 for two spheres of the same size in the limit $\epsilon \rightarrow 0$, we notice that the force and torque we obtain for squirmers of different sizes $(\alpha \neq 1$ in [18] disagree with 18 for the same system (leading to different behaviour, e.g. , for the interaction between a squirmer and a wall). We are not aware of the comparison between numerical and analytical results in such cases.

The force in the direction parallel to the line between centres, is estimated by 18 to be proportional to $\log \epsilon$ although they did not provide an explicit expression. The coefficient of $\log \epsilon$ in the force along the parallel direction to the centreline between two spheres, and the $\mathcal{O}(1)$ terms of the force in the perpendicular direction and the torque are evaluated by comparison with the numerical results of Boundary Element Method. The force in the parallel direction was estimated as $F_{z, 1} /(6 \pi \eta R)=-2.4 \log \epsilon$ [18 while we obtain from our leading order analysis that

$$
\frac{F_{z, 1}}{6 \pi \eta R}=-2 \log \epsilon
$$

from eq. (107). We also note the our expression becomes same as the analytical result of the singular term in 51 for the parallel interaction between a squirmer and a wall, that is $F_{z}$ in our notation.

We did not compute the $\mathcal{O}(1)$ terms and instead use the values of an isolated squirmer as was done in 48 for a Stokesian Dynamics simulation. This is not a bad approximation particularly for a near contact since the dominant contribution arises from the singular terms. In fact, the torque between the two squirmers moving in the same direction parallel to their centreline is numerically shown to be $\mathcal{O}(1)$ times the torque for the an isolated rotating sphere 18. Therefore, we estimate that our method is of comparable accuracy to other methods for the near-field interactions.

Finally, as briefly mentioned above, we note that the study of [18] assumed uniaxial slip flow on the surface of a squirmer. Note that this does not mean axisymmetry of the motion and rotation was assumed. In our approach, we do not need to assume axisymmetric slip flow although we have made the assumption to simplify our numerical simulations. For example, the dipolar force described by $v_{2, m}$ could have a different axis to the swimming direction described by $v_{1, m}$. In addition, the dipolar force is not necessarily uniaxial but can be biaxial. In that case, the dipolar force $v_{2, m}$ is expressed by two amplitude and three angles. As we have seen in eq. (79), the uniaxial flow is expressed by one amplitude (denoted by $v_{2}$ ) and two angles (denoted by $\alpha$ and $\beta$ ). The biaxial flow has another angle in the plane perpendicular the axis expressed by $\alpha$ and $\beta$.

So in summary, we typically find that for all the quantities calculated in 18 , we find agreement between our results and those in 18 , however in order to accurately describe the interactions between arbitrarily oriented pairs of squirmers, we need to calculate additional quantities not presented in 18 . Therefore, our method can be viewed as the minimal mathematically complete enumeration of interactions between near contact squirmers, which entails a generalization of the analytical results in [18].

Stokesian Dynamics was used in 48] to simulate collections of swimmers. However, the model used in the work is not a squirmer, which has slip boundary condition eq. (6), but rather another class of swimmer; in which the moments of force acting on the particle are imposed. With this model, it is not necessary to compute the active resistance matrix but the dynamical equation requires only the passive resistance matrix, which is known in literature.

We consider only pairwise hydrodynamic interactions, and therefore the many-body effects we observe arise from the superposition of pairwise interactions. Our method does not capture many-body interactions that are not described by the sum of pair-wise interactions. An improvement of our model could be obtained by computing the lubrication interactions between higher moments of the local velocity fields associated with each squirmer; for example, including a stresslet contribution to the resistance matrices [52,18. A recent work using pairwise interactions computes the grand mobility and resistance matrices [57] using tensorial spherical harmonics and the Galerkin method for the integral representation of the Stokes flow. This is similar to the incorporation of higher order terms of a (far field) multipole moment expansion in passive colloidal suspensions, such as performed in 52 (up to Stresslet), 58] (arbitrary order in a real space), and [59. 60. (arbitrary order in a Fourier space). However, in passive suspensions, it is known that the far field multipole moment expansion, even with higher order terms, converges slowly or does not converge at all in the limit (close proximity of particles) where lubrication interactions dominate. This was the motivation for adding the lubrication correction between all passive pairs in 52,60 using exact limiting formulas. A possible modification of the approach in [57, is to include the lubrication corrections by hand using the exact limiting formula of the active resistance matrix computed in this work and in [18], or, as proposed in [57, by numerically solving the integral equations of the boundary element method. The former method gives accurate formulas in the limit of close contact, but extending it to higher order moments might not be easy as we are not aware of systematic studies on lubrication forces as 
a function of higher moments (higher than the stresslet contribution). The latter method is straightforward, but is cumbersome as it gives a numerical table, which can be called in a many-body simulation. We stress, however that simply including the higher order far field moments themselves does not capture the lubrication forces accurately because they make singular contributions to the dynamics. Note that our formula may be expressed also by using the Cartesian tensors as demonstrated in Appendix. B

We note that standard Lattice Boltzmann simulations and Multi-Particle Collision Dynamics simulations are not designed to compute near-field interactions because of the limitation of a small mesh size and absence of ideal-gas particles to transport momentum when two particles are too close to one another. For example, in 61, the size of a particle, $R=2.3$ or $R=4.7$ in the unit of the mesh size was used and the time scale is normalised by $R / u_{0}$. Therefore, the interaction is not accurate when $\epsilon \lesssim 0.2$. Similarly, in [27, the particle size is chosen as $R=3$ in the unit of the mesh in the collision step. Therefore, the simulation has similar accuracy of the near-field interactions with that in 61. Our approach, in contrast is particularly good at short-range interactions when $\epsilon \ll 1$ because lubrication forces arise only near the contact points between two particles.

Our method, however, fails to describe intermediatedistance hydrodynamic interactions properly in contrast with the methods such as Multi-Particle Collision Dynamics and Lattice-Boltzmann Method. This may be improved by computing the higher-order terms in the matched asymptotic expansion 38.

\section{Collective behaviour}

Using the explicit expressions for the hydrodynamic interactions outlined above, we performed numerical simulations with $N$ identical particles of radius $R$ with periodic boundary conditions. We use up to $N=8192$ particles for computations including the near and far field interaction, and use up to $N=32768$ particles for the computations only with near-field interactions. Defining, the average distance between two particles, $\xi=R \sqrt{\pi / \rho_{0}}$, we vary $\xi$ from $\xi \simeq 2.65$ to $\xi \simeq 5.30$. We set $v_{1}=1$ for all swimmers and thus $u_{0} \simeq 0.32$. The size of a particle is chosen to be of unit length, thus we set $R=1$. The time scale is normalised by the time for an isolated squirmer to move a half of its body length, that is, $\tau_{0}=R / u_{0}$. There is a time scale associated with collisions, $\tau_{m}=\xi / u_{0}$. We vary the time scale from $\tau_{m} \simeq 8$ to $\tau_{m} \simeq 17$. We consider motion restricted to $2 \mathrm{D}$ but interacting via $3 \mathrm{D}$ hydrodynamic interactions as well as fully $2 \mathrm{D}$ systems. We neglect the modes with $l \geq 3$ for three-dimensional systems and $m \geq 3$ for twodimensional systems. We note that pushers $\left(v_{2}>0\right)$ and pullers $\left(v_{2}<0\right)$ having the same $\left|v_{2}\right|$ with 3D hydrodynamics are not identical when constrained to move in $2 \mathrm{D}$. This is because, defining orientations with respect to the swimming direction, the interactions at the front and the back are stronger than those at the sides. As a result, pullers, on average attract nearby objects. Pushers and pullers with $2 \mathrm{D}$ hydrodynamics are only equivalent when quadrupoles are absent, but, in general, as long as they have finite quadrupoles, they will not be identical.

Our numerical results are summarised by the phase behaviour shown in fig. 4 as a function of density $\rho_{0}=$ $\pi R^{2} N / L^{2}$ and the force dipole strength $v_{2}$. The phase diagram has been generated for systems with $N=2048$ particles. In studying the phase behaviour, we have emphasised the dependence on the sign of the force dipole $\left(v_{2} \neq 0\right)$ and contrasted them to neutral swimmers with force quadrupole and no force dipole $\left(v_{2}=0\right)$. We find significant differences between the hydrodynamic interactions with and without near-field effects. The general structure of the phase diagrams is that at low densities, the system has a disordered 'gas' state and at higher densities, there is the emergence of stable clusters except for neutral swimmers $\left(v_{2}=0\right)$. We note that the behaviour of neutral swimmers with far-field interactions only are similar to those of ABPs since there is no rotation induced by collisions. Upon including near-field effects, neutral swimmers spontaneously develop polar order. Finally, we find that stable clusters are suppressed by the near-field interactions, leading to dynamical clusters of finite size that exchange particles with bulk. The phase boundaries are qualitative; we set a specific values of threshold to distinguish between two phases. Nevertheless, as we will see in sect. 3.2, the phase boundary between the polar state and other states, for a fixed density of swimmers, are independent of threshold and easily identified. We also note in sect. 3.1 we will see there is no clear definition of phases between dynamic and static clusters.

\subsection{Static and dynamical clusters}

As the density of swimmers is increased, they collide more often with one another and possibly form aggregates (clusters) of many swimmers. We identify two classes of clusters which we denote as : static (SC) or dynamic (DC). Static clusters grow irreversibly until the majority of the swimmers are in one large cluster while dynamic clusters exchange particles with the bulk and remain of finite size. The analysis is motivated by the appearance of dynamical clusters of active particles in recent experiments 7 . 11. In two-dimensional (2D) ABP systems [10,11, 12, 62 and for squirmers confined between walls 27,63 , macroscopic phase separation has been observed associated with a clustered state. A major difference however is finite size clusters in experiments [7,11] while the infinite cluster is formed in the macroscopically phase separated state. In ABPs, rotational relaxation time is assumed to independent of a collision. Since hydrodynamic interactions result in rotation of interacting particles, this assumption is not valid for squirmers. In fact, recent simulations have shown that clusters are absent both in 2D squirmer suspensions and in a $2 \mathrm{D}$ squirmer monolayer embedded in a $3 \mathrm{D}$ fluid 28,64 . 


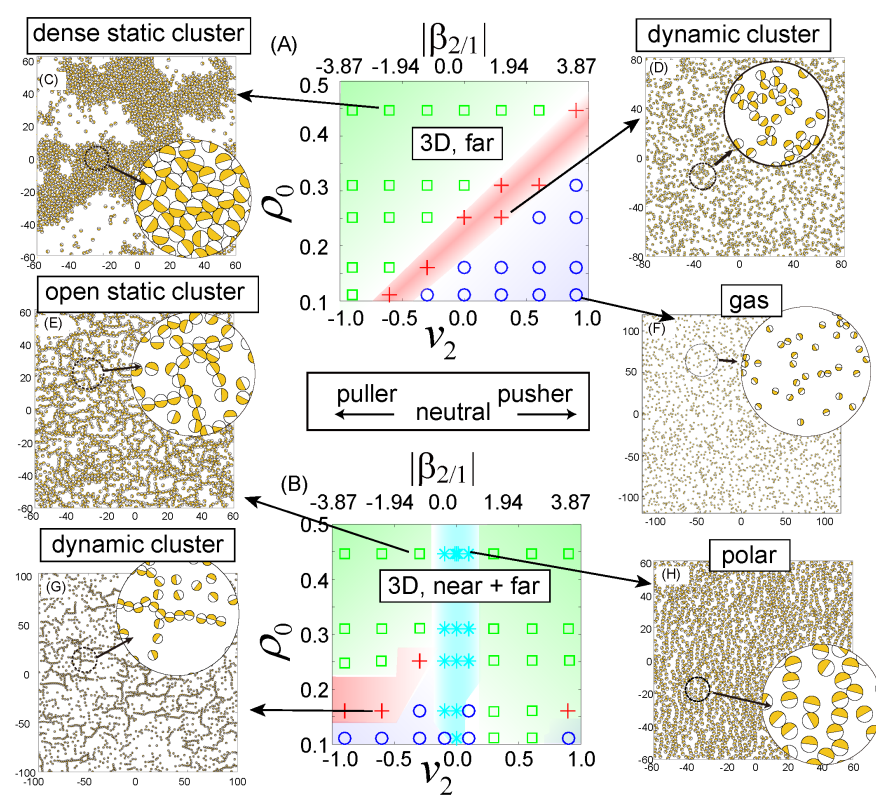

Fig. 4. (Colour Online) The state diagram of squirmers with density $\left(\rho_{0}\right)$ and dipolar strength $\left(v_{2}\right)$ with $(\mathrm{A})$ far field hydrodynamic interactions and (B) both far field and near field in 3D systems with $N=2048$ particles. Snap shots of $(\mathrm{C})$ the dense static cluster state $\left(\rho_{0}=0.447, v_{2}=-0.6\right)$, (D) the dynamic cluster state $\left(\rho_{0}=0.251, v_{2}=0.3\right)$, and $(\mathrm{F})$ the disordered state $\left(\rho_{0}=0.112, v_{2}=0.9\right)$ for a far-field-only system, and $(\mathrm{E})$ the open static cluster state $\left(\rho_{0}=0.447, v_{2}=-0.3\right),(\mathrm{G})$ the dynamic cluster state $\left(\rho_{0}=0.161, v_{2}=-0.3\right)$, and $(\mathrm{H})$ the polar state $\left(\rho_{0}=0.447, v_{2}=0.1\right)$ for a near + far system. The inset of each figure is a close view of position and orientation of particles.

Cluster formation (see fig. $5(\mathrm{~A})-(\mathrm{D})$ ) is analysed as follows. Two swimmers with centres separated by less than $r_{c}=2 R+0.01$ are in the same cluster. The $i$ th particle is labeled as $q_{i}(t)=1(0)$ when it is (not) in a cluster. We define the cluster ratio as $q(t)=(1 / N) \sum_{i=1}^{N} q_{i}(t)$. Clustered phases are defined as those with cluster ratio $\langle q\rangle>0.3$ where the average is taken over particles and time in the steady state. This threshold is chosen from the value of $\langle q\rangle$ at the maximum value of $\left\langle q^{2}\right\rangle$ when $v_{2}$ is varied under different densities. In contrast to the boundary of polar order, the boundary between gas and cluster phases is not a sharp transition but rather a smooth cross-over.

We discriminate between the clusters of the two types: static (SC) or dynamic (DC), by analysing the dynamics of particles into and out of the clusters. The variance of the clustered state $\left\langle q^{2}\right\rangle$ along the trajectory of each particle, averaged over all particles in the steady state shows how often the particles are exchanged between clustered and non-clustered states. By modeling $q_{i}(t)$ as a Telegraph process 65, these quantities are associated with on the rate of each particle joining a cluster $\left(k_{\mathrm{on}}\right)$ and leaving it $\left(k_{\text {off }}\right)$. These rates introduce time scales: the residence time inside a cluster $\tau_{\text {res }}=k_{\text {off }}^{-1}$, and the time scale to jump from one cluster to another, $\tau_{\text {out }}=k_{\text {on }}^{-1}$, in addi- tion to the time scale of a collision, $\tau_{m}$. For the Telegraph process, the mean value and its variance are given by

$$
\begin{aligned}
\langle q\rangle & =\frac{k_{\text {on }}}{k_{\text {on }}+k_{\text {off }}} \\
\left\langle q^{2}\right\rangle & =\frac{k_{\text {on }} k_{\text {off }}}{\left(k_{\text {on }}+k_{\text {off }}\right)^{2}}
\end{aligned}
$$

Therefore the ratio between the two rates is given by

$$
\frac{k_{\mathrm{on}}}{k_{\mathrm{off}}}=\frac{\langle q\rangle^{2}}{\left\langle q^{2}\right\rangle}
$$

65. The time correlation function of this process is expressed by

$$
\langle q(0) q(t)\rangle-\langle q\rangle^{2}=\left\langle q^{2}\right\rangle e^{-\left(k_{\mathrm{on}}+k_{\mathrm{off}}\right) t}
$$

From the correlation time $\left(1 /\left(k_{\text {on }}+k_{\text {off }}\right)\right)$ obtained by fitting this correlation function, we may evaluate $k_{\mathrm{on}}$ and $k_{\text {off }}$ (equivalently, $\tau_{\text {out }}$ and $\tau_{\text {res }}$ ) separately. Hence we can define the clustered states (both SC and DC) by $\tau_{\text {out }}>\tau_{m}$ in which clusters do not form even after collision. Our threshold above in terms of $\langle q\rangle$ gives the same results. At higher densities, the particles stay in clusters for longer periods, namely $k_{\text {on }} / k_{\text {off }}>1$ and $\tau_{\text {res }}>\tau_{\text {out }}$. This state is classified as the static cluster (SC). The phase boundaries between $\mathrm{SC}$ and $\mathrm{DC}$ and between DC and gas may shift under the change of the threshold of $k_{\text {on }} / k_{\text {off }}$ although we confirmed the qualitative behaviour does not depend on it.
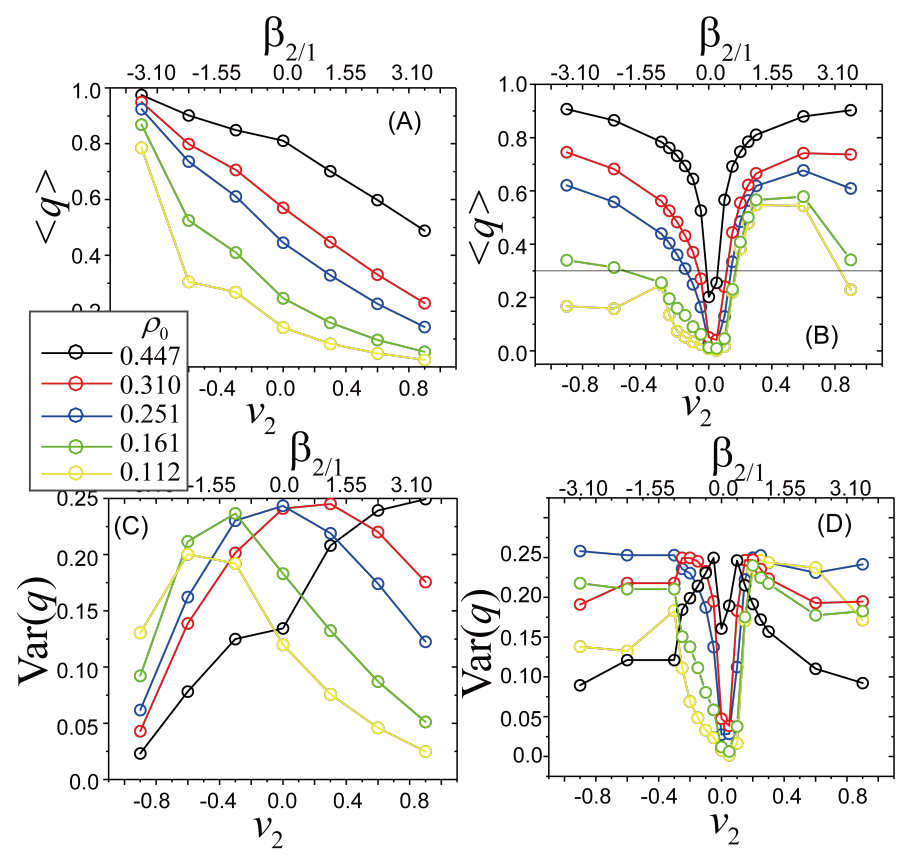

Fig. 5. (Colour Online) (A, B) The cluster ratio, $\langle q\rangle$ and $(\mathrm{C}, \mathrm{D})$ cluster fluctuation, $\left\langle q^{2}\right\rangle$ with $(\mathrm{B}, \mathrm{D})$ and without $(\mathrm{A}, \mathrm{C})$ near-field interactions as a function of dipolar strength, $v_{2}$. 


\subsection{Polar state}

In this section, we discuss the emergence of polar order and its stability. Polar order has already been reported for neutral squirmers with no force dipole in monolayer in 3D (i.e. 3D interactions, but motion in 2D) 20 and in $3 \mathrm{D}$ 66, 61, 67. It has been suggested [20 that near-field effects enhance the polar state although a weakly polar state also appears using the far-field approximation [20. This is in contrast with well established continuum arguments which demonstrate that the polar state is generically unstable for wet active systems [1,2,3. This has raised the interesting possibility of other continuum limits in these systems. Due to the expense of computational treatment of hydrodynamics however, the previous results $[20,66,61$ 67 are limited to relatively small numbers of particles, and questions remain about whether a macroscopic continuum limit had truly been achieved. Achieving this limit is one of the goals of this work.

For neutral (quadrupolar) squirmers and squirmers with small dipoles, $\left|v_{2}\right| \ll 1$, the formation of clusters is significantly suppressed and they rather self-organise into a polar state in which the swimmers align their orientations and swim in the same direction (fig. 6(A)). The polar state is robust to changes in the density but for very low density, polar order vanishes. The development of polar order is independent of initial condition. Furthermore, the amount of polar order does not change much at higher densities, but the relaxation time does become shorter. Since the far-field interaction does not cause reorientation, polar order is purely driven by near-field effects. In fact, upon screening the far-field interaction which may happen at high density 68 , we obtain almost identical polar ordered states in figs. 6(A) and (B) (also in fig. 7(D)). On introducing rotational noise, the disorder-polar transition is still present but occurs at a higher density.

In the phase diagram (see fig. 4), the polar state corresponds to $\langle p\rangle=(1 / N)\left|\sum_{i} \mathbf{p}^{(i)}\right|>0.7$. This value is chosen from the value of $\langle p\rangle$ above the transition from the gas state to the polar state induced either by pusher dipole force or the rotational noise (see fig. 7(A,B) and fig. 9). We emphasize that this stringent criterion means that the polar regions of the phase (state) diagram correspond to strongly ordered globally polar states.

The polar state is present for small but nonzero $v_{2}$, but as $v_{2}$ is increased, polar order vanishes as shown in figs. 7(A), (C) and (D). This is due to large fluctuations of the angular velocity (see fig. $6(\mathrm{C})$ ), $\left\langle\omega^{2}\right\rangle \sim v_{2}^{2}$ from eq. 29). For pushers $\left(v_{2}>0\right)$, polar order disappears around $v_{2} \simeq 0.15\left(\beta_{2 / 1} \simeq 0.58\right)$. The transition from the polar-orderd state to a non-polar state is accompanied by divergence of fluctuations of the polar order as shown in fig. $7(\mathrm{~B})$. On the other hand, pullers $\left(v_{2}<0\right)$ behave somewhat differently as one increases $\left|v_{2}\right|$. First, for pullers, the amount of polar order fluctuates more than for pushers, and accordingly the mean polar order parameter $\langle p\rangle$ becomes noisy. Second, we do not observe a distinct peak in the fluctuations in polarity between the polar order and the disorder state. Nevertheless, when the density is high ( $\rho_{0}=0.447$ and $\xi=2.65$ ), there is clearer divergence of fluctuation as shown in fig. 7(D). At the transition point, there is phase separation between polar order and disorder. For large enough $\left|v_{2}\right|$, polar order completely vanishes for both types of swimmers. The polar order still present even when the far-field interactions are switched off. The critical dipolar force is slightly larger for such systems for pushers, but the polar order is dependent on the dipolar force $v_{2}$ similar to the near and far field interactions (fig. 7(A)). For pullers, the near field interactions also suppress polar order while the noisy behaviours discussed above vanish in this case (fig. $7(\mathrm{C})$ ) suggesting that it arises from the far-field interactions.

The polar order is stable even for very large numbers of particles. In fig. 8, polar order is shown as a function of the number of particles, $N$ up to $N=8192$ for the near+far field system and $N=32768$ for the near-field-only system. Polar order survives even for large system sizes, and therefore we conclude that the system is asymptotically in a state with macroscopic global polar order. The simulations without far-field interactions give slightly larger stronger polar order for the same density. Nevertheless, the systems with and without far-field interactions show the same behaviour. The mean cluster ratio of the polar phase is nearly zero throughout the range of system sizes, indicating that there is no clustering associated with these polar states.

\subsection{Orientational fluctuations}

To examine the stability of polar order to fluctuations, we add uniformly distributed white noise, customarily used in the Vicsek model 69, to the angular velocity of each swimmer. To be precise, position and orientation are updated by eqs. (1) and (2) as before. However the angular velocity defined eq. 29 now has an additional fluctuating component and becomes

$$
\begin{aligned}
\boldsymbol{\omega}^{(i)} & =\sum_{j \neq i, l, m} \omega_{l m}^{(j)} \boldsymbol{\Phi}_{l m}^{(j i)}+\boldsymbol{\omega}_{f}^{(i)}(t) \\
\left\langle\boldsymbol{\omega}_{f}^{(i)}(t)\right\rangle & =0,\left\langle\boldsymbol{\omega}_{f}^{(i)}(t) \cdot \boldsymbol{\omega}_{f}^{(i)}\left(t^{\prime}\right)\right\rangle=\frac{\sigma^{2}}{3} \delta_{i j} \delta\left(t-t^{\prime}\right)
\end{aligned}
$$

for a system with fixed density $\rho_{0}$. We emphasize that no noise is added to the translational velocity, eq. (28) and any additional fluctuations in position arise purely from rotational noise, eq. (67). In our quasi-2D system, rotation induced by the noise occurs only in the plane. The amount of polar order as a function of the amplitude $\sigma^{2}$ of the rotational noise is shown in fig. 9 . Around $\sigma=\sigma_{c} \simeq 0.13$, we observe a phase transition between a polar state and a gas state at $\rho_{0}=0.16$. The polar state is present for lower noise amplitudes while the gas state is stable at higher noise amplitudes. The critical noise amplitude, $\sigma_{c}$ is density dependent. At higher densities of squirmers, $\left(\rho_{0}=0.44\right)$, the transition from the polar state to the gas state occurs at higher noise amplitudes. For both densities studied $\left(\rho_{0}=0.16,0.44\right)$, the polar ordered state has a uniform density with no observable micro-phase separation. The fluctuations of the polarity 

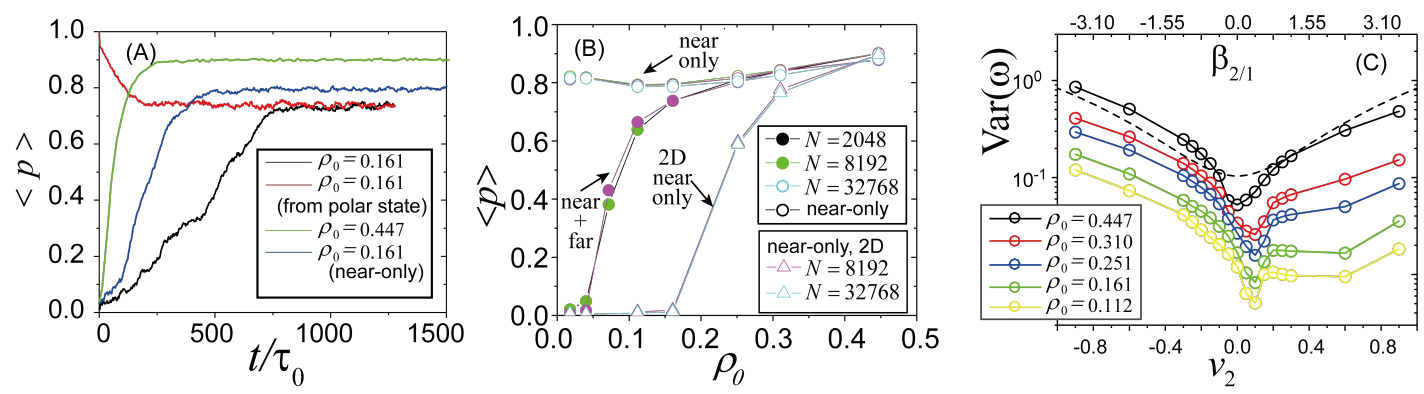

Fig. 6. (Colour Online) (A) Time evolution of $\langle p\rangle$ for neutral swimmers at $\rho_{0}=0.16(\xi=4.43)$ shown in black (the initial condition is disordered state) and red (ordered state) and $\rho_{0}=0.447(\xi=2.65)$ shown in green. The polar order of the nearfield-only system is shown in blue. (B) the mean polarity, $\langle p\rangle$ for neutral swimmers $v_{2}=0$ vs density, $\rho_{0}$. The solid (open) circles correspond to the simulations with (without) the far-field interaction in 3D systems. The open triangles correspond to the results of the near-field interaction in $2 \mathrm{D}$ systems. Both include the near field interaction. (C) rotational fluctuation, $\left\langle\omega^{2}\right\rangle$ vs dipolar strength, $v_{2}$. The dashed line shows $\left\langle\omega^{2}\right\rangle \sim v_{2}^{2}$ for the guide for the eyes.
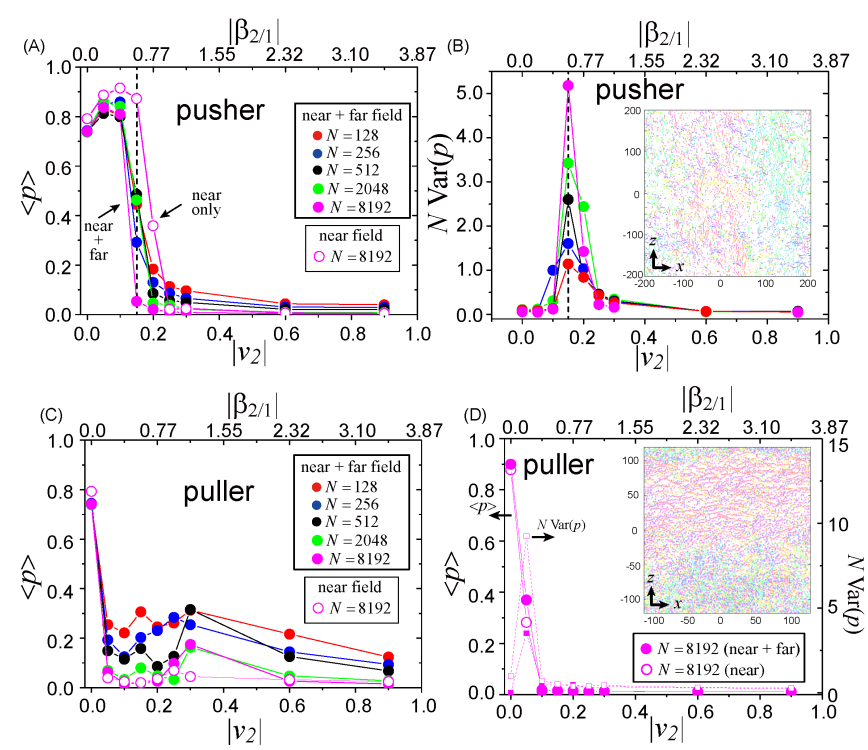

Fig. 7. (Colour Online) The mean polarity as a function of the dipolar slip flow $v_{2}$ on a squirmer at $\rho_{0}=0.16(\xi=4.43)$. The corresponding values of the squirmer index $\beta_{2 / 1}$ is shown at the upper axis. The polar order $\langle p\rangle$ for pushers (A) and pullers $(\mathrm{C})$. The near and far field systems are shown by the filled circles while the systems only with near field interaction is shown by the open circles. (B) Fluctuation for pushers. The inset shows a snapshot at $v_{2}=0.15$ for $N=8192$. The colour shows orientation angle of each particle from $z$ axis (red: $0,2 \pi$, green: $2 \pi / 3$, and blue: $4 \pi / 3$ ). (D) The mean polarity and fluctuations about the mean for pullers at $\rho_{0}=0.447$ $(\xi=2.65)$. Both the results of the near+far (solid circle) and the near-field-only (open circle) systems are shown. Fluctuation for pullers is shown by squares. The inset shows a snapshot at $v_{2}=-0.05$ for $N=8192$.

about its mean value diverges as the amplitude of noise approaches its critical value $\sigma_{c}$. Interestingly, a polar band appears near the critical point, in which the low density gas state and high density polar state coexist. This be-
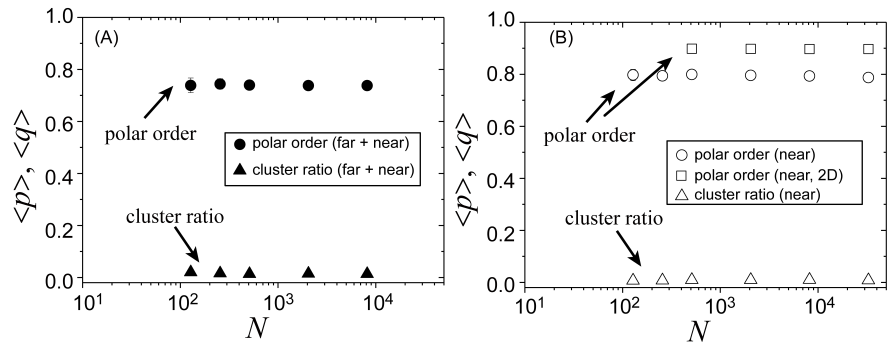

Fig. 8. (Colour Online) The system size dependence of the mean polar order $\langle p\rangle$ and the mean cluster ratio $\langle q\rangle$ at $\rho_{0}=$ $0.16(\xi=4.43)$ of the neutral swimmer $v_{2}=0$ for $(\mathrm{A})$ far- and near-field systems and for (B) near-field-only systems. in (B), the results of two-dimensional neutral squirmers for $\rho_{0}=0.447$ $(\xi=2.65)$ are also shown in the open triangles.

haviour is similar to the gas-polar phase transition of the Vicsek-type models (fig. $9(\mathrm{C})$ ).

We characterise the rotational noise by a rotational diffusion coefficient $D_{r}$, or equivalently a rotational diffusion time, $\tau_{r}$

$$
\tau_{r}=\frac{1}{2 D_{r}}=\frac{3}{\sigma^{2}}
$$

The rotational diffusion time at the critical noise is $\tau_{r}=$ $3 / \sigma_{c}^{2} \simeq 178$. We can define a rotational Péclet number,

$$
\mathrm{Pe}=\frac{u_{0} \tau_{r}}{R}
$$

which compares the self-propulsion speed and rotational diffusion. At the critical noise, $\mathrm{Pe} \simeq 60$ for $\rho_{0}=0.16$ and $\mathrm{Pe} \simeq 10$ for $\rho_{0}=0.44$. Although the rotational Péclet number for $\sigma \ll \sigma_{c}$ for the higher density is in the region where macroscopic phase separation has been observed for active brownian particles [70, in our system we find a uniform density of squirmers except in the vicinity of the transition from the polar to gas phase where the noise amplitude has its critical value, $\sigma_{c}$ (Fig. fig.noise(C)). Note, however, the rotational Péclet number eq. (69) is only 

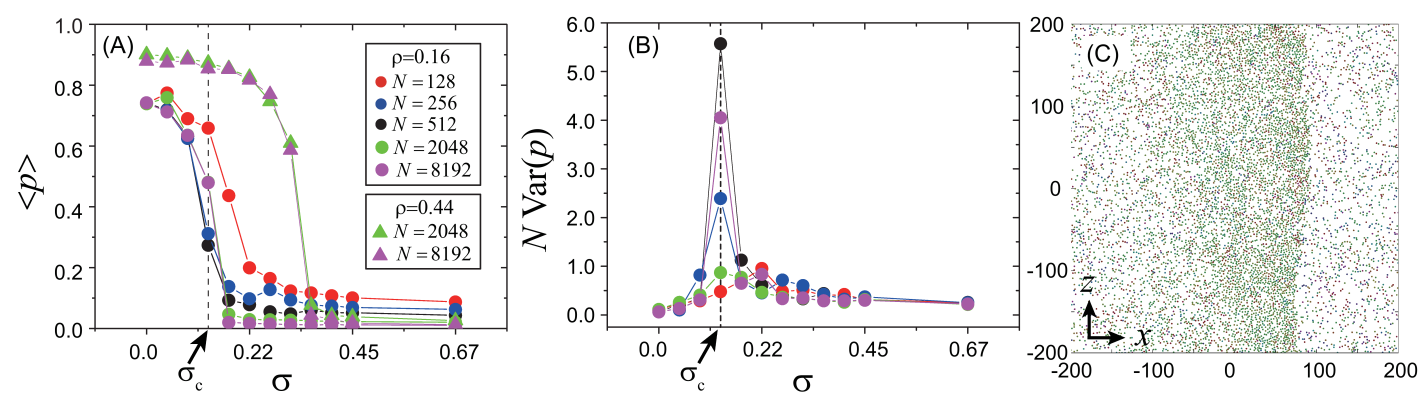

Fig. 9. (Colour Online) (A) The mean polarity as a function of orientational noise amplitude at $\rho_{0}=0.16(\xi=4.43$, closed circles) and at $\rho_{0}=0.447\left(\xi=2.65\right.$, closed triangles) for the neutral swimmers $\left(v_{2}=0\right.$ and $v_{1}=1.0$ in eq. (30p). (B) The variance of the polarity. The dashed line corresponds to the critical point where $\sigma_{c}=0.13$ for $\rho_{0}=0.16$. (C) The polar band state near the critical amplitude of noise for $N=8192$ for $\rho_{0}=0.16$. The colour shows orientation angle of each particle from $z$ axis (red: $0,2 \pi$, green: $2 \pi / 3$, and blue: $4 \pi / 3$ ).

based on the noise added to the system, and does not take account of additional rotational fluctuations generated by collisions, which occur even in deterministic squimers (see fig. 6(C)). This is consistent with previous results that suggest the suppression of macroscopic phase separation in squirmer suspensions [28].

\subsection{Collective behaviours of two-dimensional squirmers}

We also carried out simulations with 2D hydrodynamic interactions using the method outlined in sect 2.3. The polar state also appears here although it occurs at higher densities than the system with 3D hydrodynamics as shown in fig. 10(B). At intermediate densities, dynamic clusters appear. This is in agreement with [71] which showed a gap between the dimension of the interaction and the dimension of the system led to a weaker suppression of alignment. Similar to 3D systems, the far-field interaction does not lead to polar order even for neutral swimmers as shown in fig. 10(A). In this case, static clusters appear at higher densities while the system is disordered at low densities. The near-field interaction results in polar order for neutral swimmers.

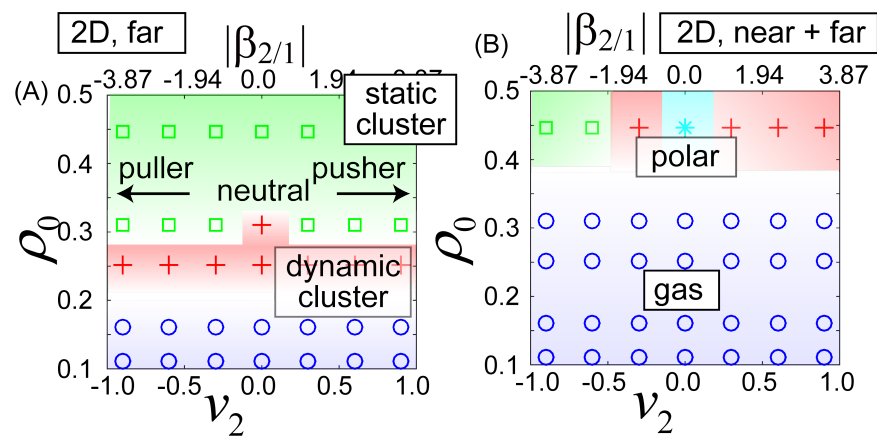

Fig. 10. (Colour Online) The state diagram of squirmers with density $\left(\rho_{0}\right)$ and dipolar strength $\left(v_{2}\right)$ with $(\mathrm{A})$ far field hydrodynamic interactions and (B) both far field and near field in 2D systems with $N=512$ particles.
Swimmers only with the near-field interaction also show polar order (fig. 6(B)). In contrast to 3D systems, polar order only appears when the density is high. The emergence of polar order is insensitive to system size and polar order is present for the largest system size simulated: $N=32768$ particles. This is analogous to what was found in $3 \mathrm{D}$ systems.

\section{Swimmer collisions and polar order}

\subsection{Two-body collisions}

In this section, we investigate the two body interaction in detail to get insights into the emergence of collective behaviour leading to polar order. Figure 11 shows some trajectories of two 'colliding' squirmers, whose initial orientation would lead to them approaching other in the absence of interactions. The hydrodynamic (lubrication and farfield) interactions lead to a reorientation of the swimmers which eventually move in separate directions. We call this process a 'collision'. If the separation between the initial angles is the same as the separation between the final angles then the collision is said to be symmetric. The neutral swimmer has symmetric collisions when the initial angle is small while there is a small asymmetry that develops when the initial angle becomes large, leading to smaller angular separations after collisions. The final angles of the pusher (puller) are larger (smaller) than the initial angles. As the initial angle increases, neutral swimmers develop bound states in which two particles stay a close distance (fig. 11(A)) from each other for an extended period during which lubrication interactions dominate before eventually separating. These bound states are the source of the alignment and development of polar order observed. This is because the bound states are robust, i.e. remain stable even after additional collisions with other swimmers while the other post collision states are unstable to future collisions.

In order to quantify the dynamics of collisions, we consider trajectories with reflection symmetry about the $x y$ plane shown in fig. 12(A). The position and orientation 
of the two particles are expressed by $\beta_{1}=\pi / 2+\phi$ and $\beta_{2}=\pi / 2-\phi, x^{(1)}=x^{(2)}$, and $h_{12}=\left(z^{(1)}-z^{(2)}\right) / 2$. Due to the near-field interaction, alignment and transient bound states appear as shown in fig. 12(B) and (C). For swimmer separations where near-field effects dominate, the orientational dynamics is

$$
\dot{\phi}=-g_{1} \cos \phi+g_{2} \sin 2 \phi
$$

with $g_{1} \sim u_{0} / R$ and $g_{2} \sim v_{2} / R$. A particle with incident angle $\phi_{0}$ at the outer boundary of the near field region at $t=0$ rotates so that $\phi(\tau)=0$ at the collision time $t=\tau$. For the neutral swimmer $g_{2}=0$, we have $g_{1} \tau=\log (1-$ $\tan \phi) /(1+\tan \phi)$. The trajectory is shown in fig. $12(\mathrm{C}))$. When the initial angle is large enough, a neutral swimmer spends a long time bound to another.

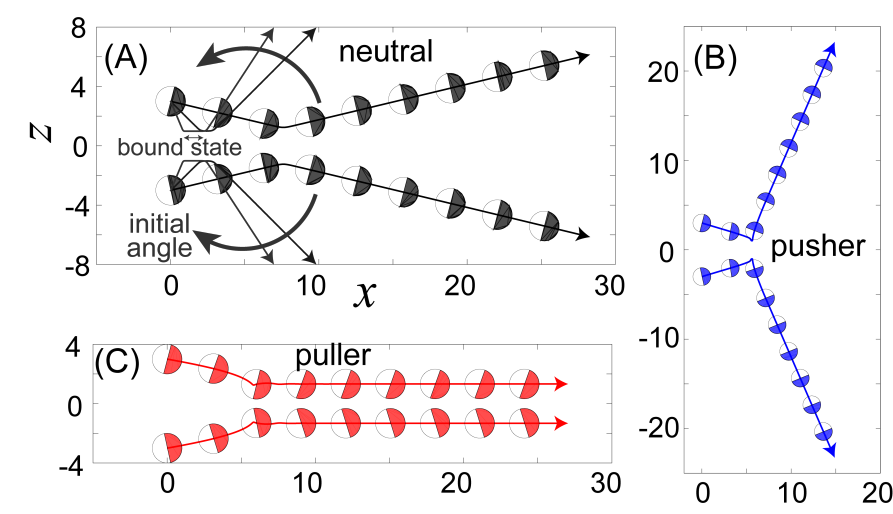

Fig. 11. (Colour Online) Trajectories for (A) neutral $\left(v_{2}=0\right)$, (B) pusher $\left(v_{2}=0.9\right)$, and (C) puller $\left(v_{2}=-0.9\right)$ swimmers. For the neutral swimmers, the trajectories with larger initial angles are also shown. The directions of the trajectories are shown by arrows.

For the far-field-only system, two pushers $\left(v_{2}>0\right)$ align while two pullers $\left(v_{2}<0\right)$ stick to each other. However, these states are unstable to rotational fluctuations induced by the fact that a swimmer typically experiences collisions with many particles along its trajectory. Hence the system does not develop any polar order. The nearfield interaction leads to alignment and transient bound states as shown in fig. 12(B) and (C). Neutral swimmers $\left(v_{2}=0\right)$, have the longest residence times and the weakest rotational fluctuations due to the absence of rotations from far field interactions. For small incident angles $\left(\phi_{0}\right)$, the reflection angle $\left(\phi_{f}\right)$ increases linearly with incidence angle, $\phi_{f} \simeq \phi_{0}$, leading to symmetric collisions. However as the incidence angle becomes larger, the reflection angle no longer increases with the incident angle and we observe a saturation of the reflection angle at a value $\left|\phi_{s}\right| \lesssim \pi / 4$ as shown in fig. $12(\mathrm{C})$. This asymmetry between incident and reflection angles $\left(\left\langle\phi_{f}\right\rangle\left\langle\left\langle\phi_{0}\right\rangle\right.\right.$, see fig. 12(C)) is what leads eventually to alignment. Similar behaviour is seen for pushers and pullers, however, shorter residence times and stronger rotational fluctuations destroy the polar state for both of them.
In simulations, the details of this process depend weakly on the contact interaction; the choice of the interaction potential leads only to slight shifts of saturation angles. Nevertheless, our conclusions about the collective behaviours and phase boundaries are independent of the choice of potential. We have used the truncated Lennard-Jones repulsive interaction as well as other types of interaction, e.g. eq. (53). They all result in qualitatively the same behaviour with a slightly shifted saturation angle and boundaries between different types of collective behaviour. We note that the saturation angle $\left(\left|\phi_{s}\right| \approx \pi / 4\right)$ in fig. 12 (E) originates from direct contacts between squirmers via repulsive forces from the interaction potential. Without the repulsive interaction, eq. 70 implies that incident and final angles are the same as shown in fig. $12(\mathrm{~F})$. This suggests that the polar order we observe for neutral and near neutral swimmers is induced by saturation of a final angle with respect to an incident angle, which arises from the loss of its memory of an incident angle due to the contact interaction.
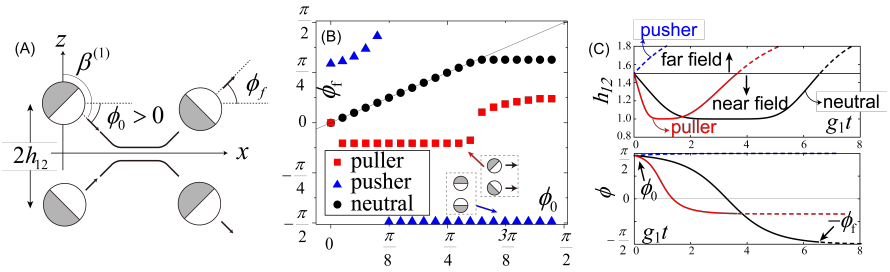

Fig. 12. (Colour Online) (A) Schematics of two-body collisions. (B) The incidence $\phi_{0}$ and reflection $\phi_{f}$ angles for symmetric collisions. The solid line shows $\phi_{0}=\phi_{f}$. The negative $\phi_{f}$ corresponds to the bound states schematically drawn in the insets. (C) The dynamics of the separation $h_{12}(t)$ and the angle $\phi(t)$ in the near-field region obtained from eq. 46 for $g_{2} / g_{1}=1$. Motion outside the near-field region is indicated by dashed lines.

\subsection{Many-body effects}

Although our model includes only pairwise interactions, combining them with each other, results in many-body effects which become relevant for a non-dilute suspension. In fact, for a dense suspension where $\xi-2 \lesssim 1$, the interaction is dominated by the lubrication interaction between two swimmers which is well approximated by a sum of two-body interactions.

To understand how these many-body effects give rise to collective behaviour, we have carried out numerical simulations of a Vicsek-style model, in which the interactions in the angular direction (fig. 13(A)). The interaction is given by

$$
\omega^{(i)}=\frac{1}{2} \sum_{j \neq i}\left[\sin \left(\beta^{(i)}-\theta_{j i}\right)+\sin \left(\beta^{(j)}-\theta_{j i}\right)\right]
$$

We are able to reproduce the same polar-disorder phase transition by increasing the amplitude of noise as in fig. 13 (B). 
Therefore, the detailed form of the hydrodynamic interactions in the translational direction given by eq. (28) are not essential for the development of polar order, and only the effect of the hydrodynamic interactions on the orientational dynamics in eq. (29) are necessary. One critical difference with the conventional Vicsek model is that here, the excluded volume interactions are necessary to generate polar order; without them the system remains the gas state even at lower values of the noise amplitude and with strong rotational interactions. Polar order appears irrespective of the choice of repulsive interactions. Here in order to see the effect of excluded volume interaction, we use the repulsive interaction as $\mathbf{F}_{i j}=V_{\text {int }}\left(r_{i j}-2 R\right) \hat{\mathbf{r}}_{i j}$.
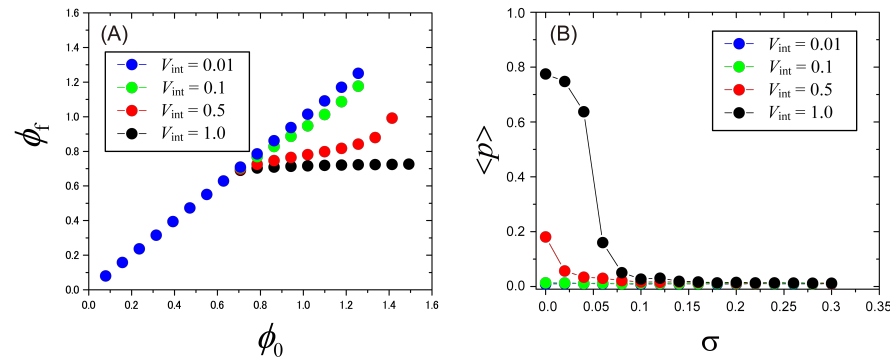

Fig. 13. (Colour Online) (A) Incident and the final angles of two-body collisions between two swimmers described by the model with the interaction eq. (71). (B) The mean polar order $\langle p\rangle$ as a function of the strength of noise $\sigma$ at $N=8192$ and $\rho_{0}=0.16(\xi=4.43)$ corresponding to $(\mathrm{A})$.

\subsection{Hydrodynamic argument}

The existence of polar order is fundamentally surprising because of the apparent contradiction with the well accepted generic instability of polar/nematic order of wet active matter 72,1. An explanation of how this is possible can be found using a simple argument based on a generalized hydrodynamic description of the conserved quantities and spontaneously broken continuous symmetries of the system [1. It is noteworthy that it emphasizes the importance of collisions of the swimmers with each other as essential for the formation of polar order.

We begin by constructing a two-fluid model for the system, for the suspending fluid (volume fraction, $1-\phi$ ) and the active particle (squirmer) 'network' (volume fraction $\phi)$. We define a local displacement variable $\mathbf{u}(\mathbf{r}, t)$ for the active particle network which is related to the density variations of squirmers. The Newtonian fluid it is suspended in is characterised by a velocity field $\mathbf{v}(\mathbf{r}, t)$. Finally we identify a local polar order parameter, $\mathbf{p}(\mathbf{r}, t)$. An isolated squirmer swims with velocity $u_{0} \mathbf{p}$ relative to the background fluid. The fluid obeys equation,

$$
\rho_{f} \dot{\mathbf{v}}=\eta \nabla^{2} \mathbf{v}-\nabla P+\nabla \cdot \boldsymbol{\sigma}^{a}-\mathbf{f}^{c}
$$

where $\rho_{f}=(1-\phi) \rho$ is the average density of fluid, $\mathbf{f}^{c} \propto \rho_{a}^{2}$ is the force on a squirmer due to collisions with others, where $\rho_{a}=\phi \rho$ is the average density of active particles. The fluid is taken as incompressible, $\nabla \cdot \mathbf{v}=0$. The active stress $\boldsymbol{\sigma}^{a}$ is given by $\sigma_{i j}^{a}=\nu p_{i} p_{j}$, where $\nu \propto v_{2}$.

The active 'network' has an equation of motion which indicates that the velocity, $\dot{\mathbf{u}}(\mathbf{r}, t)$ of the active particles at $\mathbf{r}$ is given by

$$
\dot{\mathbf{u}}(\mathbf{r}, t)=\mathbf{v}(\mathbf{r}, t)+u_{0} \mathbf{p}(\mathbf{r}, t)-\mathbf{F} / \zeta
$$

where $\mathbf{F}(\mathbf{r}, t)$ is the total force density on the active particles at point $\mathbf{r}$, and $\zeta$ is the slope of the force-velocity curve of the squirmer, i.e. in the absence of external forces (here due to collisions), the squirmers move with speed $u_{0}$.

In addition there is the equation of motion for the polar director

$$
\dot{\mathbf{p}}=-u_{0} \mathbf{p} \cdot \nabla \mathbf{p}+\boldsymbol{\omega} \cdot \mathbf{p}+\gamma \mathbf{e} \cdot \mathbf{p}+K \nabla^{2} \mathbf{p}+\cdots
$$

where $\omega_{i j}=\frac{1}{2}\left(\partial_{i} v_{j}-\partial_{j} v_{i}\right), e_{i j}=\frac{1}{2}\left(\partial_{i} v_{j}+\partial_{j} v_{i}\right)$ and $K$ is an effective Frank elastic constant that assigns a cost to distortions of the local polar order and in general will include non-equilibrium contributions [1,73.

It is illustrative to consider the system at low density as $\rho_{a} \rightarrow 0$. Then we find $\mathbf{f}^{c}=0$ and performing a linearised analysis about the homogeneous state, $\dot{\mathbf{u}}=\dot{\mathbf{u}}_{0}, \mathbf{p}=\mathbf{p}_{0}$, $\mathbf{v}_{0}=\dot{\mathbf{u}}_{0}-u_{0} \mathbf{p}_{0}$ we obtain the generic instability of the polar state [72.

Now let us switch on $\mathbf{f}^{c} \neq 0$. The force $\mathbf{f}^{c}$ leads to slowing down of the swimmer, taking eq. (73) above when gradients of $\mathbf{u}$ vanish :

$$
\mathbf{f}^{c}=\zeta\left(\dot{\mathbf{u}}-\mathbf{v}-u_{0} \mathbf{p}\right) \quad \Rightarrow \quad \dot{\mathbf{u}}=\mathbf{v}+u_{0} \mathbf{p}+\mathbf{f}^{c} / \zeta,
$$

i.e. when $\mathbf{f}^{c}=0$, the squirmer swims at its free speed.

We can replace $\mathbf{f}^{c}$ by $\zeta\left(\dot{\mathbf{u}}-\mathbf{v}-u_{0} \mathbf{p}\right)$ in eq. 72$)$. So now performing the linearised expansion $\mathbf{p}=\mathbf{p}_{0}+\delta \mathbf{p}(\mathbf{r}, t), \mathbf{v}=$ $\mathbf{v}_{0}+\delta \mathbf{v}(\mathbf{r}, t), \boldsymbol{\omega}=\boldsymbol{\omega}_{0}+\delta \boldsymbol{\omega}(\mathbf{r}, t), \mathbf{e}=\mathbf{e}_{0}+\delta \mathbf{e}(\mathbf{r}, t)$ around $\mathbf{v}_{0}=\dot{\mathbf{u}}_{0}-u_{0} \mathbf{p}_{0}$,

$$
\begin{aligned}
0 & =\eta \nabla^{2} \delta \mathbf{v}+\zeta \delta \mathbf{v}-\nabla P+\nabla \cdot \boldsymbol{\sigma}^{a} ; \nabla \cdot \delta \mathbf{v}=0 \\
\partial_{t} \delta \mathbf{p} & =-u_{0} \mathbf{p}_{0} \cdot \nabla \delta \mathbf{p}+\delta \boldsymbol{\omega} \cdot \mathbf{p}_{0}+\gamma \delta \mathbf{e} \cdot \mathbf{p}_{0}+K \nabla^{2} \delta \mathbf{p}
\end{aligned}
$$

with a finite screening length $\xi \sim \sqrt{\eta / \zeta}$ which weakens the generic instability from a long-wavelength to a finite wavelength instability and stabilizes the polar state on long lengthscales. Hence a comparison between the screening length $\xi$ and the active lengthscale, $\sqrt{K /|\nu|}[74$, allows us to determine the onset of polar order for $|\nu|<\nu_{c}=K / \xi^{2}$, i.e. for swimmers that are close to neutral. In this longwavelength limit, the appearance of polar order is symmetric about $\nu=0$. However, short range hydrodynamics corresponding to higher-order terms in spatial gradients can easily break that symmetry.

\section{Discussion and summary}

In summary, we have re-analyzed the collective behaviour of self-propelled particles (squirmers) taking account of the effects of hydrodynamics when the swimmers are close 
to one another. We find the existence of a polar ordered phase whose stability is dominated by short-range interactions arising from lubrication forces between two squirmers. The global phase separation of active particles with repulsive interactions, is suppressed by hydrodynamic interactions and we observe instead dynamic clusters of finite size for a large range of intermediate densities. We see gel-like extended state at high enough densities.

We emphasize that our goal is to develop an approximate method of dealing with hydrodynamics of squirmers, that can efficiently simulate large enough numbers of swimmers to capture accurately the macroscopic behaviour of wet active matter. Polar order has been observed in earlier simulations with hydrodynamics, but due to the computationally expensive nature of fluid mechanical calculations, these were limited by small numbers of particles and it remained an open question if the observed phenomena were simply finite size effects. Due to limitations in computational power, there is a trade-off that must be made between how accurately the hydrodynamic flow field is resolved and increasing the number of particles. For Navier-Stokes solvers like Lattice Boltzmann or Multi-Particle Collision Dynamics, this is typically done by limiting the resolution of the flow field to a scale comparable to the squirmer size so that the behaviour of particles in very close proximity is not treated accurately; such models are best for particles at intermediate separations. Here we develop an alternative approach with different strengths; developing a description that is very accurate when the particles are very close and very far apart but which performs less well for intermediate separations. A further strength of our method is the ability to switch of different contributions to the motion to identify the dominant mechanisms behind each macroscopic phenomena. With this model we are able to simulate large numbers of particles and confirm that the qualitative behaviour seen by small simulations remains even for much larger systems.

Nevertheless, we do find some quantitative differences with the previous numerical results. Of particular interest is the transition induced by puller slip flow (negative $v_{2}$ ), namely it has been reported that the amount of polar order for pullers near the neutral swimmer $\left(v_{2}=0\right)$ is larger than that of pushers which our results do not show. Interestingly, in our simulations, see fig. 7(A,C), we find an asymmetry in the appearance of polar order about $v_{2}=0$; that is, the amount of polar order observed for pushers $\left(v_{2}>0\right)$ is different to pullers $\left(v_{2}<0\right)$. For pushers, peak polarity is slightly shifted from $v_{2}=0$, and the amount of polar order decays rapidly to zero beyond a critical force dipole magnitude. For pullers, the polar order first decays rapidly to $\langle p\rangle \simeq 0.2$, then decays slowly to zero as $v_{2}$ increases, particularly for smaller systems. This slow decay does not appear in the near-field-only systems. This indicates the importance of the far-field hydrodynamics, and also implies that the polar order for pullers at intermediate values of $v_{2}$ is influenced by medium-scale hydrodynamic interactions, which are only crudely approximated within our approach. It has also been reported that a density wave appears for pullers [61. We have seen this in the purely two-dimensional system (2D with $2 \mathrm{D}$ interactions) and quasi-two-dimensional system (2D with $3 \mathrm{D}$ interactions) with noise, but not for pullers in the quasitwo-dimensional system (2D with $3 \mathrm{D}$ interactions). We emphasize however that, since each of the methods used so far has disadvantages, either small number of particles, inaccurate treatment of the near-field interactions, or intermediate range interactions; that further work is needed to clarify some of these issues. We may speculate however that the behaviour above seen using the Lattice Boltzmann Method to simulate pullers is dominated by intermediate-ranged, many-body hydrodynamic interactions, which are not well captured by our method.

Since our treatment of hydrodynamics is approximate, particularly for intermediate distances between swimmers, a detailed comparison with high resolution full hydrodynamic simulations is required in the future. In this direction, it would be useful to investigate the effect of lubrication forces on the simulation techniques accurate for intermediate to long separation between squirmers, such as the Lattice Boltzmann Method and Multi-Particle Collision Dynamics. On the other hand, it would also be interesting to include intermediate-distance interactions more accurately in our model by using particle-mesh-type simulations, which has been used in particle simulations with electrostatic interactions 75$]$.

\section{Acknowledgments}

The authors are grateful to S. Fielding, T. Ishikawa and R. Golestanian for helpful discussions. NY acknowledges the support by JSPS KAKENHI Grant Numbers JP16H00793 and $17 \mathrm{~K} 05605$. TBL is supported by BrisSynBio, a BBSRC/EPSRC Advanced Synthetic Biology Research Center (grant number BB/L01386X/1). We would like to thank the Isaac Newton Institute for Mathematical Sciences, Cambridge, for support and hospitality during the programmes, "The Mathematics of Liquid Crystals" and "Dynamics of active suspensions, gels, cells and tissues" where work on this article was started.

\section{Author contribution statement}

NY and TBL developed the theoretical model. NY performed the simulations. Both authors interpreted the results and wrote the paper.

\section{A Coefficients of vector spherical harmonics}

Since the expansion of the slip velocity in terms of the vector spherical harmonics is carried out with the $z$-axis in a (fixed) laboratory frame, the coefficient of each spherical harmonic in the slip velocity $v_{l m}$ varies with rotation of the particle. There is a one-to-one map from the angles 
to the coefficients $\{\alpha, \beta\} \mapsto v_{l m}$. When $v_{l m}$ has only the first, $l=1$ mode, we may define a vector $\mathbf{p}$ with components $v_{1,-1}, v_{1,0}$, and $v_{1,1}$. The second mode, $l=2$ can be represented by a second-rank tensor, with higher modes correspond to higher-rank tensors [76]. In terms of spherical harmonics, these are given by $(2 l+1)$-dimensional vectors. Here $v_{l m}=\left(v_{l,-l}, \cdots v_{l, 0}, \cdots, v_{l, l}\right)$ is expressed by the polar angle $\mathbf{p}^{(i)}=(\cos \alpha \sin \beta, \sin \alpha \sin \beta, \cos \beta)$. as

$$
v_{1, m}^{(i)}=v_{1}^{(i)}\left(\begin{array}{c}
\frac{1}{\sqrt{2}} \sin \beta^{(i)} e^{i \alpha^{(i)}} \\
\cos \beta^{(i)} \\
-\frac{1}{\sqrt{2}} \sin \beta^{(i)} e^{-i \alpha^{(i)}}
\end{array}\right)
$$

and

$$
v_{2, m}^{(i)}=v_{2}^{(i)}\left(\begin{array}{c}
\frac{\sqrt{6}}{4} \sin ^{2} \beta^{(i)} e^{2 i \alpha^{(i)}} \\
\sqrt{\frac{3}{2}} \sin \beta \cos \beta^{(i)} e^{i \alpha^{(i)}} \\
-\sqrt{\frac{3}{2}} \cos ^{2} \beta^{(i)}-\frac{1}{2} \\
\frac{\sqrt{6}}{4} \sin ^{2} \beta^{(i)} \cos \beta^{(i)} e^{-i \alpha^{(i)}}
\end{array}\right) .
$$

\section{B Cartesian tensors}

While we have written out our flow fields in terms of vector spherical harmonics, they may equivalently be expressed in terms of Cartesian tensors. To do this, we define the intrinsic velocity $\mathbf{U}_{0}$, the intrinsic angular velocity, and the intrinsic strain flow arising from slip velocity, eq. (6) by

$$
\begin{aligned}
& \mathbf{U}_{0}=-\frac{1}{4 \pi R^{2}} \int \mathbf{v}_{s} d S \\
& \mathbf{\Omega}_{0}=-\frac{3}{8 \pi R^{3}} \int \mathbf{n} \times \mathbf{v}_{s} d S \\
& \mathbf{E}_{0}=-\frac{3}{8 \pi R} \int\left[\mathbf{n v}_{s}+\mathbf{v}_{s} \mathbf{n}\right] d S
\end{aligned}
$$

where $\mathbf{n}(\theta, \varphi)$ is a normal vector pointing outward a spherical surface. In terms of the coefficients in eq. (6), these quantities are expressed as

$$
\begin{aligned}
& \mathbf{U}_{0}=-\frac{1}{4 \pi} 4 \sqrt{\frac{\pi}{3}}\left(\begin{array}{c}
\frac{v_{1,-1}-v_{1,1}}{\sqrt{2}} \\
-i \frac{v_{1,-1}+v_{1,1}}{\sqrt{2}} \\
v_{1,0}
\end{array}\right) \\
& \boldsymbol{\Omega}_{0}=\frac{3}{8 \pi R} 4 \sqrt{\frac{\pi}{3}}\left(\begin{array}{c}
\frac{w_{1,-1}-w_{1,1}}{\sqrt{2}} \\
-i \frac{w_{1,-1}+w_{1,1}}{\sqrt{2}} \\
w_{1,0}
\end{array}\right)
\end{aligned}
$$

$$
\begin{aligned}
& v_{2, \pm 2}=v_{2,2}+v_{2,-2} \\
& \tilde{v}_{2, \pm 2}=v_{2,2}-v_{2,-2} \\
& v_{2, \pm 1}=v_{2,1}+v_{2,-1} \\
& \tilde{v}_{2, \pm 1}=v_{2,1}-v_{2,-1}
\end{aligned}
$$

Using the orientation of a swimmer, $\mathbf{p}$, in eq. (11), the intrinsic velocities are expressed as

$$
\begin{aligned}
& \mathbf{U}_{0}=-\frac{v_{1}}{\sqrt{3 \pi}} \mathbf{p} \\
& \mathbf{\Omega}_{0}=-\frac{w_{1}}{2} \sqrt{\frac{3}{\pi}} \mathbf{p} \\
& \mathbf{E}_{0}=-\frac{3 R v_{2}}{4} \sqrt{\frac{3}{\pi}}\left(\mathbf{p p}-\frac{1}{3} \mathbf{I}\right)
\end{aligned}
$$

The physical meaning of these quantities is clear from Faxen's laws. The force, torque, and stress for a fixed isolated swimmer is expressed as

$$
\begin{aligned}
& \mathbf{F}^{(a)}=-6 \pi \eta R \mathbf{U}_{0} \\
& \mathbf{T}^{(a)}=-8 \pi \eta R^{3} \boldsymbol{\Omega}_{0} \\
& \mathbf{S}^{(a)}=-\frac{20}{3} \pi \eta R^{3} \mathbf{E}_{0} .
\end{aligned}
$$

These are active force, torque, and stress discussed in sect. 2.2. Note that the active stress, $\mathbf{S}^{(a)}$ does not lead to motion and rotation of an isolated swimmer.

We will rewrite the velocity field in Cartesian coordinates and the intrinsic flow, $\mathbf{U}_{0}, \boldsymbol{\Omega}_{0}$, and $\mathbf{E}_{0}$ described above. We expand the velocity field in terms of the modes $l$ as

$$
\mathbf{v}(\mathbf{r})=\sum_{l=1}^{\infty} \mathbf{v}_{l}(\mathbf{r})
$$

The first mode is

$$
\begin{aligned}
\mathbf{v}_{1}(\mathbf{r}) & =\left(\frac{R}{r}\right)^{3}\left[\mathbf{U}_{0} \cdot \mathbf{n n}-\frac{1}{2}\left(\mathbf{U}_{0} \cdot \mathbf{t t}+\mathbf{U}_{0} \cdot \mathbf{b b}\right)\right] \\
& =\left(\frac{R}{r}\right)^{3}\left[\frac{3}{2} \mathbf{U}_{0} \cdot \mathbf{n n}-\frac{1}{2} \mathbf{U}_{0}\right] .
\end{aligned}
$$

The second mode is given by

$\frac{\mathbf{E}_{0}}{-\frac{3 R}{8 \pi} 4 \sqrt{\frac{3 \pi}{10}}}=\left(\begin{array}{ccc}v_{2, \pm 2}-\sqrt{\frac{2}{3}} v_{2,0} & i \tilde{v}_{2, \pm 2} & -\tilde{v}_{2, \pm 1} \\ i \tilde{v}_{2, \pm 2} & -v_{2, \pm 2}-\sqrt{\frac{2}{3}} v_{2,0} & -i v_{2, \pm 1} \\ -\tilde{v}_{2, \pm 1} & -i v_{2, \pm 1} & 2 \sqrt{\frac{2}{3}} v_{2,0}\end{array}\right)+\left(\frac{R}{r}\right)^{4}\left(\mathbf{E}_{0} \odot_{2} \mathbf{t t t}+\mathbf{E}_{0} \odot_{2} \mathbf{b b b}\right)$

$$
\begin{gathered}
\mathbf{v}_{2}(\mathbf{r})=3\left[\left(\frac{R}{r}\right)^{2}-\left(\frac{R}{r}\right)^{4}\right] \mathbf{E}_{0} \odot_{2} \mathbf{n n n} \\
+\left(\frac{R}{r}\right)^{4}\left(\mathbf{E}_{0} \odot_{2} \mathbf{t t t}+\mathbf{E}_{0} \odot_{2} \mathbf{b b}\right) \\
\mathbf{A} \odot_{2} \mathbf{B}=A_{i j} B_{i j} .
\end{gathered}
$$




\section{Axisymmetric motion in 3D}

First we consider the motion in $z$-direction. In polar coordinates, the velocity field is described using the stream function as

$$
\mathbf{v}=\left(\frac{1}{r} \frac{\partial \psi}{\partial z}, 0,-\frac{1}{r} \frac{\partial \psi}{\partial r}\right)
$$

The stream function satisfies the biharmonic-like equation

$$
E^{2} E^{2} \psi=0
$$

with the operator $E^{2}=\frac{\partial^{2}}{\partial r^{2}}-\frac{1}{r} \frac{\partial}{\partial r}+\frac{\partial^{2}}{\partial z^{2}}$. We expand the stream function in $\epsilon$ as

$$
\psi=\epsilon \psi_{0}+\epsilon^{2} \psi_{1}+\cdots
$$

with the operator also expanded as

$$
E^{2}=\frac{1}{\epsilon^{2}} \frac{\partial^{2}}{\partial \tilde{Z}^{2}}+\cdots
$$

For the near field, the velocity fields are at the lowest order $v_{r} \sim \mathcal{O}\left(\epsilon^{-1 / 2}\right)$ and $v_{z} \sim \mathcal{O}\left(\epsilon^{0}\right)$.

The passive force acting on the sphere given a translational velocity $u_{z}$ is 77,45

$$
\begin{aligned}
& \frac{F_{z 1}^{(p)}}{6 \pi \eta R} \simeq-\frac{1}{4 \epsilon}\left(u_{z}^{(i)}-u_{z}^{(j)}\right)-\frac{1}{2}\left(u_{z}^{(i)}+u_{z}^{(j)}\right) \mathcal{I}_{z}^{(p)} \\
& \frac{F_{z 2}^{(p)}}{6 \pi \eta R} \simeq \frac{1}{4 \epsilon}\left(u_{z}^{(i)}-u_{z}^{(j)}\right)-\frac{1}{2}\left(u_{z}^{(i)}+u_{z}^{(j)}\right) \mathcal{I}_{z}^{(p)}
\end{aligned}
$$

Here we have two terms; one is a singular term obtained from asymptotic analysis of the problem (b), and the other term is $\mathcal{O}\left(\epsilon^{0}\right)$, which corresponds to the problem (f) in fig. 3. Extending the matched asymptotic expansion, the calculation of the $\mathcal{O}\left(\epsilon^{0}\right)$ term may be possible. However, instead, the patching procedure is often used; $\mathcal{O}\left(\epsilon^{0}\right)$ term is replaced by the value when two sphere is touching each other 78. This value could be obtained either from numerical results or from the analytical calculation using tangent-sphere coordinates with some truncation. In fact, the unknown constant $\mathcal{I}_{z}^{(p)}$ is computed as $\mathcal{I}_{z}^{(p)}=\mathcal{O}\left(\epsilon^{0}\right) \simeq$ 0.645141 . It may also be possible to replace the force acting on the two particles moving together approximately by that of the isolated particle. Then we set $\mathcal{I}_{z}^{(p)}=1$.

For the active force, we expand the stream function instead as eq. 102

$$
\psi=\epsilon^{2} \psi_{0}+\epsilon^{3} \psi_{1}+\cdots
$$

which is appropriate for the boundary conditions in the active problem, $v_{r} \sim \mathcal{O}(\sqrt{\epsilon})$ and $v_{z} \sim \mathcal{O}(\epsilon)$. The force acting on the sphere is computed as

$$
\begin{aligned}
\frac{F_{z 1}^{(a)}}{6 \pi \eta R} & \simeq-\frac{1}{2} \log \epsilon \sum_{l} \mathcal{N}_{l, 0} V_{l, 0}+\frac{1}{3} \mathcal{I}_{z}^{(a)} \mathcal{N}_{1,0} W_{1,0} \\
\frac{F_{z 2}^{(a)}}{6 \pi \eta R} & \simeq \frac{1}{2} \log \epsilon \sum_{l} \mathcal{N}_{l, 0} V_{l, 0}+\frac{1}{3} \mathcal{I}_{z}^{(a)} \mathcal{N}_{1,0} W_{1,0}
\end{aligned}
$$

where as in the main text, we have used

$$
\begin{aligned}
V_{l m} & =\frac{l(l+1)}{2}\left((-1)^{l} v_{l m}^{(i)}+v_{l m}^{(j)}\right) \\
W_{l m} & =\frac{l(l+1)}{2}\left((-1)^{l} v_{l m}^{(i)}-v_{l m}^{(j)}\right) .
\end{aligned}
$$

We simply replace this force by the force required to fix an isolated squirmer by adding an unknown constant $\mathcal{I}_{z}^{(a)} \sim$ $\mathcal{O}\left(\epsilon^{0}\right)$.

\section{Non-axisymmetric motion and rotation in 3D}

When the spheres move with translational velocity in $x$ direction and with angular velocity in $y$-direction, the velocity field is expressed in polar coordinates as

$$
\mathbf{v}=(U \cos \varphi, V \sin \varphi, W \cos \varphi) .
$$

The boundary conditions are rewritten as

$$
\begin{gathered}
U\left(\tilde{Z}=H_{i}\right)=U_{H_{i}} \\
V\left(\tilde{Z}=H_{i}\right)=V_{H_{i}} \\
W\left(\tilde{Z}=H_{i}\right)=W_{H_{i}},
\end{gathered}
$$

where the subscripts $i=1,2$ correspond to Sphere (i) and $(\mathrm{j})$, respectively, in fig. 2. The velocity on the surface is not necessarily constant as for rigid translational and rotational motion, but is dependent on $\tilde{\rho}$. The expansion discussed in the next section assumes the boundary conditions satisfy $U_{H_{1}} \sim \mathcal{O}\left(\epsilon^{0}\right), \quad U_{H_{2}} \sim \mathcal{O}\left(\epsilon^{0}\right), \quad V_{H_{1}} \sim$ $\mathcal{O}\left(\epsilon^{0}\right), \quad V_{H_{2}} \sim \mathcal{O}\left(\epsilon^{0}\right), \quad W_{H_{1}} \sim \mathcal{O}\left(\epsilon^{1 / 2}\right), \quad W_{H_{2}} \sim \mathcal{O}\left(\epsilon^{1 / 2}\right)$. Following [50], the velocity fields are expanded in $\epsilon$ as

$$
\begin{aligned}
P(r, z) & =\epsilon^{-3 / 2} P_{0}(\tilde{\rho}, \tilde{Z})+\mathcal{O}\left(\epsilon^{-1 / 2}\right) \\
U(r, z) & =U_{0}(\tilde{\rho}, \tilde{Z})+\mathcal{O}(\epsilon) \\
V(r, z) & =V_{0}(\tilde{\rho}, \tilde{Z})+\mathcal{O}(\epsilon) \\
W(r, z) & =\epsilon^{1 / 2} W_{0}(\tilde{\rho}, \tilde{Z})+\mathcal{O}\left(\epsilon^{3 / 2}\right) .
\end{aligned}
$$

At lowest order in $\epsilon$, the Stokes equation becomes $[50$.

$$
\begin{aligned}
\frac{\partial P_{0}}{\partial \tilde{\rho}} & =\frac{\partial^{2} U_{0}}{\partial \tilde{Z}^{2}} \\
-\frac{P_{0}}{\tilde{\rho}} & =\frac{\partial^{2} V_{0}}{\partial \tilde{Z}^{2}} \\
\frac{\partial P_{0}}{\partial \tilde{Z}} & =0 .
\end{aligned}
$$

The solution of $U_{0}$ and $V_{0}$ is expressed by $P_{0}(\tilde{\rho})$ and the condition of incompressibility leads to the following Reynolds equation

$$
\tilde{\rho}^{2} P_{0}^{\prime \prime}+C[\lambda, \tilde{\rho}] P_{0}^{\prime}-P_{0}+D[\lambda, \tilde{\rho}]=0
$$

where $C[\lambda, \tilde{\rho}]$ and $D[\lambda, \tilde{\rho}]$ are functions of $\tilde{\rho}$ with the parameter $\lambda$ and the boundary conditions eq. $(112)$. The 
parameter $\lambda$ is dependent on the curvature of the second particle (See fig. 2). For pairs of particles of the same size, $\lambda=-1$.

The relative motion gives singular terms arising from passive problems (a), (c), and (g). The passive problem (g) has only a singular torque and its force is $\mathcal{O}\left(\epsilon^{0}\right)$. As in the previous section, we evaluate the $\mathcal{O}\left(\epsilon^{0}\right)$ terms using the passive problems (e) and (g). The passive force and torque on each sphere are

$$
\begin{aligned}
\frac{F_{1}^{(p)}}{6 \pi \eta R} \simeq & \frac{1}{6} \log \epsilon\left[\left(u_{x}^{(1)}-u_{x}^{(2)}\right)-R\left(\omega_{y}^{(1)}+\omega_{y}^{(2)}\right)\right] \\
& -\frac{1}{2}\left(u_{x}^{(1)}+u_{x}^{(2)}\right) \mathcal{I}_{x}^{(p)}-\frac{R}{2}\left(\omega_{y}^{(1)}-\omega_{y}^{(2)}\right) \mathcal{J}_{x}^{(p)} \\
\frac{F_{2}^{(p)}}{6 \pi \eta R} \simeq & \frac{1}{6} \log \epsilon\left[-\left(u_{x}^{(1)}-u_{x}^{(2)}\right)+R\left(\omega_{y}^{(1)}+\omega_{y}^{(2)}\right)\right] \\
& -\frac{1}{2}\left(u_{x}^{(1)}+u_{x}^{(2)}\right) \mathcal{I}_{x}^{(p)}-\frac{R}{2}\left(\omega_{y}^{(1)}-\omega_{y}^{(2)}\right) \mathcal{J}_{x}^{(p)} \\
\frac{T_{1}^{(p)}}{8 \pi \eta R^{2}} \simeq & \frac{1}{8} \log \epsilon\left[-\left(u_{x}^{(1)}-u_{x}^{(2)}\right)+\frac{R}{10}\left(4 \omega_{y}^{(1)}+\omega_{y}^{(2)}\right)\right. \\
& \left.+\frac{3 R}{5}\left(\omega_{y}^{(1)}-\omega_{y}^{(2)}\right)\right]-\frac{1}{2}\left(u_{x}^{(1)}+u_{x}^{(2)}\right) \mathcal{G}_{x}^{(p)} \\
\frac{T_{2}^{(p)}}{8 \pi \eta R^{2}} & \frac{1}{8} \log \epsilon\left[-\left(u_{x}^{(1)}-u_{x}^{(2)}\right)+\frac{R}{10}\left(\omega_{y}^{(1)}+4 \omega_{y}^{(2)}\right)\right. \\
& \left.-\frac{3 R}{5}\left(\omega_{y}^{(1)}-\omega_{y}^{(2)}\right)\right]-\frac{1}{2}\left(u_{x}^{(1)}+u_{x}^{(2)}\right) \mathcal{G}_{x}^{(p)} .
\end{aligned}
$$

Here the constant is known for the passive problem (e) and (g) as $\mathcal{I}_{x}^{(p)} \simeq 0.72426, \mathcal{G}_{x}^{(p)} \simeq 0.11843$, and $\mathcal{J}_{x}^{(p)} \simeq 0.15802$.

For the active problem, the force generated by the slip velocity is

$$
\begin{aligned}
\frac{F_{1}^{(a)}}{6 \pi \eta R} \simeq & \frac{1}{6} \log \epsilon \sum_{l, m= \pm 1} m \mathcal{N}_{l|m|} V_{l m} \\
& -\frac{1}{3} \sum_{m= \pm 1} m \mathcal{N}_{1,|m|} W_{1, m} \mathcal{I}_{x}^{(a)} \\
\frac{F_{2}^{(a)}}{6 \pi \eta R} \simeq & -\frac{1}{6} \log \epsilon \sum_{l, m= \pm 1} m \mathcal{N}_{l|m|} V_{l m} \\
& -\frac{1}{3} \sum_{m= \pm 1} m \mathcal{N}_{1,|m|} W_{1, m} \mathcal{I}_{x}^{(a)} \\
\frac{T_{1}^{(a)}}{8 \pi \eta R^{2}} \simeq & -\frac{1}{20} \log \epsilon \sum_{l, m= \pm 1} m \mathcal{N}_{l|m|}\left(\frac{5}{2} V_{l m}+\frac{3}{2} W_{l m}\right) \\
\frac{T_{2}^{(a)}}{8 \pi \eta R^{2}} \simeq & -\frac{1}{20} \log \epsilon \sum_{l, m= \pm 1} m \mathcal{N}_{l|m|}\left(\frac{5}{2} V_{l m}-\frac{3}{2} W_{l m}\right) .
\end{aligned}
$$

The $\mathcal{O}\left(\epsilon^{0}\right)$ terms (the second line in the expression for the forces) are added assuming that these forces are similar to those of an isolated squirmer with a coefficient $\mathcal{I}_{x}^{(a)}$.

\section{E Two Dimensions}

\section{E.1 Isolated squirmer}

The solution of the Stokes equation is decomposed into the radial $\mathbf{r}$ and the tangential (t) directions

$$
\mathbf{v}=v_{r} \mathbf{r}+v_{t} \mathbf{t}
$$

where by defining

$$
\begin{gathered}
\mathbf{v}_{m}=\left(v_{s, m},-\tilde{v}_{s, m}\right)=v_{m}(\cos m \beta, \sin m \beta) \\
v_{r}=\mathbf{u} \cdot \mathbf{r}\left(\frac{R}{r}\right)^{2}+\sum_{m=2}^{\infty}\left[\left(\frac{R}{r}\right)^{m-1}-\left(\frac{R}{r}\right)^{m+1}\right] \frac{m}{2} \mathbf{v}_{m} \cdot \mathbf{r}_{m} \\
v_{t}=-\mathbf{u} \cdot \mathbf{t}\left(\frac{R}{r}\right)^{2} \\
-\sum_{m=2}^{\infty}\left[\frac{m-2}{2}\left(\frac{R}{r}\right)^{m-1}-\frac{m}{2}\left(\frac{R}{r}\right)^{m+1}\right] \mathbf{v}_{m} \cdot \mathbf{t}_{m}
\end{gathered}
$$

with

$$
\begin{aligned}
& \mathbf{r}_{m}=(\cos m \theta, \sin m \theta) \\
& \mathbf{t}_{m}=(-\sin m \theta, \cos m \theta) .
\end{aligned}
$$

We may define the polarity of the particle

$$
\mathbf{p}=(\cos \beta, \sin \beta)
$$

then we may rewrite as

$$
\mathbf{u}=u \mathbf{p}
$$

\section{E.2 Far-field interaction}

As in the three dimensional case, we use Faxen's laws to evaluate the far-field interaction. Since we are working in a force-free system, the leading order term should be $\mathbf{u}=$ $\mathbf{v}_{0}+\mathcal{O}\left(\nabla^{2} \mathbf{v}_{0}\right)$. The motion of one particle perturbed by a second particle is

$$
\begin{aligned}
\mathbf{u}^{(i)}= & u \mathbf{p}^{(i)}+\sum_{j \neq i}\left(\frac{R}{r_{i j}}\right)^{2}\left[\left(\mathbf{u}^{(j)} \cdot \hat{\mathbf{r}}_{i j}\right) \hat{\mathbf{r}}_{i j}-\left(\mathbf{u}^{(j)} \cdot \hat{\mathbf{t}}_{i j}\right) \hat{\mathbf{t}}_{i j}\right] \\
& +\sum_{j} \frac{R}{2 r_{i j}}\left(\mathbf{v}_{2}^{(j)} \cdot \hat{\mathbf{r}}_{i j, 2}\right) \hat{\mathbf{r}}_{i j}
\end{aligned}
$$

where

$$
\begin{aligned}
\hat{\mathbf{r}}_{i j} & =\frac{\mathbf{r}^{(i)}-\mathbf{r}^{(j)}}{r_{i j}}=\left(\cos \theta_{i j}, \sin \theta_{i j}\right) \\
\hat{\mathbf{r}}_{i j, m} & =\left(\cos m \theta_{i j}, \sin m \theta_{i j}\right)
\end{aligned}
$$


and the unit tangent vector is

$$
\hat{\mathbf{t}}_{i j}=\left(-\sin \theta_{i j}, \cos \theta_{i j}\right) .
$$

We may define the generalized tangent vector as

$$
\hat{\mathbf{t}}_{i j, m}=\left(-\sin m \theta_{i j}, \cos m \theta_{i j}\right),
$$

these vectors are transformed by $\theta_{i j} \leftrightarrow \theta_{j i}=\theta_{i j}+\pi$ as $\mathbf{r}_{i j, m}=(-1)^{m} \mathbf{r}_{j i, m}$ and $\mathbf{t}_{i j, m}=(-1)^{m} \mathbf{t}_{j i, m}$. The angular velocity is

$$
\omega^{(i)}=-\frac{1}{4} \sum_{j \neq i}\left(\mathbf{v}_{m}^{(j)} \cdot \hat{\mathbf{t}}_{i j}\right) \frac{R}{r_{i j}^{2}} \mathbf{e}_{z}
$$

Note that even in two dimensions, there is no contribution from the $m=1$ mode on the rotation, because the flow field of the $m=1$ mode is in fact the same as potential flow. The explicit forms in eqs. (57) and (58)

$$
\begin{aligned}
u_{\|, 1} & =\left(\frac{R}{r_{i j}}\right)^{2}\left(\mathbf{u}^{(j)} \cdot \hat{\mathbf{r}}_{i j}\right) \\
u_{\perp, 1} & =-\left(\frac{R}{r_{i j}}\right)^{2}\left(\mathbf{u}^{(j)} \cdot \hat{\mathbf{t}}_{i j}\right) \\
u_{\|, 2} & =\frac{R}{2 r_{i j}}\left(\mathbf{v}_{2}^{(j)} \cdot \hat{\mathbf{r}}_{i j, 2}\right) \hat{\mathbf{r}}_{i j} \\
\omega_{2} & =-\frac{1}{4}\left(\mathbf{v}_{2}^{(j)} \cdot \hat{\mathbf{t}}_{i j}\right) \frac{R}{r_{i j}^{2}}
\end{aligned}
$$

\section{E.3 Near-field interaction}

The lubrication theory of a cylinder approaching toward a wall or another cylinder was discussed in 79, 80. The analysis was first done using bipolar coordinates 79 It has been further extended to the flow around a cylinder in a confined space 81,82 . In this section, we will use the same stretched coordinates as three-dimensional flow.

We may divide the motion into components of the parallel and perpendicular directions to the center line. In stretched coordinates, the slip velocity is

$$
\mathbf{v}_{s}^{(i)}=v_{s}^{(i)} \mathbf{t}^{(i)},
$$

which is expanded in $\epsilon$ in a similar fashion to the three dimensional case. The superscript $i=1,2$ denotes the sphere 1 or 2 . In stretched coordinates, we expand the velocity field and pressure. As in the three-dimensional case, the velocity and angular velocity are calculated from the force-free and torque-free conditions. From the linearity of the problem, we may decompose $u_{y}$ and $\left(u_{x}, \omega\right)$. By rotating the result, the velocity and angular velocity in general coordinates are given by

$$
\begin{aligned}
\mathbf{u}^{(i)} & =u_{\perp} \hat{\mathbf{t}}_{j i}+u_{\|} \hat{\mathbf{r}}_{j i} \\
\omega^{(i)}= & -\frac{1}{4}\left(\mathbf{u}_{0}^{(i)}-\mathbf{u}_{0}^{(j)}\right) \cdot \hat{\mathbf{t}}_{j i} \\
& +\frac{1}{4} \sum_{m}\left(3(-1)^{m-1} \mathbf{v}_{s, m}^{(i)}+\mathbf{v}_{s, m}^{(j)}\right) \cdot \hat{\mathbf{t}}_{j i, m} .
\end{aligned}
$$

Here we define the unit vectors with $\mathbf{r}_{21}=\mathbf{r}_{2}-\mathbf{r}_{1}$. The concrete form is

$$
\begin{aligned}
u_{\|}= & \frac{1}{2}\left(\mathbf{u}_{0}^{(i)}+\mathbf{u}_{0}^{(j)}\right) \cdot \hat{\mathbf{r}}_{j i} \\
& -\frac{1}{2} \sqrt{\epsilon} \sum_{m} m\left((-1)^{m} \mathbf{v}_{s, m}^{(i)}+\mathbf{v}_{s, m}^{(j)}\right) \cdot \hat{\mathbf{r}}_{j i, m} \\
u_{\perp}= & \frac{1}{4}\left(3 \mathbf{u}_{0}^{(i)}+\mathbf{u}_{0}^{(j)}\right) \cdot \hat{\mathbf{t}}_{j i} \\
& -\frac{1}{4} \sum_{m}\left((-1)^{m} \mathbf{v}_{s, m}^{(i)}+\mathbf{v}_{s, m}^{(j)}\right) \cdot \hat{\mathbf{t}}_{j i, m} .
\end{aligned}
$$

The explicit forms in eqs. (57) and $(58)$ are as follows:

$$
\begin{gathered}
u_{\|, 1}=\frac{1}{2}\left(\mathbf{u}_{0}^{(i)}+\mathbf{u}_{0}^{(j)}\right) \cdot \hat{\mathbf{r}}_{j i}-\frac{1}{2} \sqrt{\epsilon}\left(-\mathbf{v}_{s, 1}^{(i)}+\mathbf{v}_{s, 1}^{(j)}\right) \cdot \hat{\mathbf{r}}_{j i, 1} \\
u_{\|, m}=-\frac{1}{2} \sqrt{\epsilon} m\left((-1)^{m} \mathbf{v}_{s, m}^{(i)}+\mathbf{v}_{s, m}^{(j)}\right) \cdot \hat{\mathbf{r}}_{j i, m} \\
u_{\perp, 1}=\frac{1}{4}\left(3 \mathbf{u}_{0}^{(i)}+\mathbf{u}_{0}^{(j)}\right) \cdot \hat{\mathbf{t}}_{j i}-\frac{1}{4}\left(-\mathbf{v}_{s, 1}^{(i)}+\mathbf{v}_{s, 1}^{(j)}\right) \cdot \hat{\mathbf{t}}_{j i, 1} \\
u_{\perp, m}=-\frac{1}{4}\left((-1)^{m} \mathbf{v}_{s, m}^{(i)}+\mathbf{v}_{s, m}^{(j)}\right) \cdot \hat{\mathbf{t}}_{j i, m} \\
\omega_{1}=-\frac{1}{4}\left(\mathbf{u}_{0}^{(i)}-\mathbf{u}_{0}^{(j)}\right) \cdot \hat{\mathbf{t}}_{j i}+\frac{1}{4}\left(3 \mathbf{v}_{s, 1}^{(i)}+\mathbf{v}_{s, 1}^{(j)}\right) \cdot \hat{\mathbf{t}}_{j i, 1} \\
\omega_{m}=\frac{1}{4}\left(3(-1)^{m-1} \mathbf{v}_{s, m}^{(i)}+\mathbf{v}_{s, m}^{(j)}\right) \cdot \hat{\mathbf{t}}_{j i, m} .
\end{gathered}
$$

\section{References}

1. M. C. Marchetti, J. F. Joanny, S. Ramaswamy, T. B. Liverpool, J. Prost, Madan Rao, and R. Aditi Simha. Hydrodynamics of soft active matter. Rev. Mod. Phys., 85:11431189, 2013.

2. J. Toner, Y. Tu, and S. Ramaswamy. Hydrodynamics and phases of flocks. Ann. Phys., 318(1):170-244, 2005.

3. Sriram Ramaswamy. The mechanics and statistics of active matter. Ann. Rev. Cond. Mat. Phys., 1:323-345, 2010.

4. Tamás Vicsek, András Czirók, Eshel Ben-Jacob, Inon Cohen, and Ofer Shochet. Novel type of phase transition in a system of self-driven particles. Phys. Rev. Lett., 75(6):1226-1229, 1995.

5. Henricus H. Wensink, Jörn Dunkel, Sebastian Heidenreich, Knut Drescher, Raymond E. Goldstein, Hartmut Löwen, and Julia M. Yeomans. Meso-scale turbulence in living fluids. Proc. Nat. Acad. Sci., 109(36):14308-14313, 2012.

6. M E Cates. Diffusive transport without detailed balance in motile bacteria: does microbiology need statistical physics? Rep. Prog. Phys., 75(4):042601, 2012.

7. Jeremie Palacci, Stefano Sacanna, Asher Preska Steinberg, David J. Pine, and Paul M. Chaikin. Living crystals of light-activated colloidal surfers. Science, 339(6122):936940, 2013.

8. Antoine Bricard, Jean-Baptiste Caussin, Nicolas Desreumaux, Olivier Dauchot, and Denis Bartolo. Emergence of macroscopic directed motion in populations of motile colloids. Nature, 503(7474):95-98, 2013. 
9. Eric Bertin, Hugues Chaté, Francesco Ginelli, Shradha Mishra, Anton Peshkov, and Sriram Ramaswamy. Mesoscopic theory for fluctuating active nematics. New Journal of Physics, 15(8):085032, 2013.

10. Yaouen Fily and M. Cristina Marchetti. Athermal phase separation of self-propelled particles with no alignment. Phys. Rev. Lett., 108:235702, 2012.

11. Ivo Buttinoni, Julian Bialké, Felix Kümmel, Hartmut Löwen, Clemens Bechinger, and Thomas Speck. Dynamical clustering and phase separation in suspensions of selfpropelled colloidal particles. Phys. Rev. Lett., 110:238301, 2013.

12. Gabriel S. Redner, Michael F. Hagan, and Aparna Baskaran. Structure and dynamics of a phase-separating active colloidal fluid. Phys. Rev. Lett., 110:055701, 2013.

13. Thomas Speck. Collective behavior of active brownian particles: From microscopic clustering to macroscopic phase separation. The European Physical Journal Special Topics, 225(11):2287-2299, 2016.

14. M. J. Lighthill. On the squirming motion of nearly spherical deformable bodies through liquids at very small reynolds numbers. Communications on Pure and Applied Mathematics, 5(2):109-118, 1952.

15. JR Blake. Self propulsion due to oscillations on the surface of a cylinder at low reynolds number. Bulletin of the Australian Mathematical Society, 5(02):255-264, 1971.

16. Ramin Golestanian, Tanniemola B. Liverpool, and Armand Ajdari. Propulsion of a molecular machine by asymmetric distribution of reaction products. Physical Review Letters, 94(22):220801, 2005.

17. Y. Ibrahim and T.B. Liverpool. How walls affect the dynamics of self-phoretic microswimmers. The European Physical Journal Special Topics, 225(8):1843-1874, Oct 2016.

18. T. Ishikawa, MP Simmonds, and TJ Pedley. Hydrodynamic interaction of two swimming model microorganisms. J. Fluid Mech., 568:119-160, 2006.

19. I. Llopis and I. Pagonabarraga. Dynamic regimes of hydrodynamically coupled self-propelling particles. Eur. Phys. Lett., 75(6):999, 2006.

20. Takuji Ishikawa, J. T. Locsei, and T. J. Pedley. Development of coherent structures in concentrated suspensions of swimming model micro-organisms. J. Fluid Mech., 615(1):401-431, 2008.

21. Kenta Ishimoto and Eamonn A. Gaffney. Squirmer dynamics near a boundary. Phys. Rev. E, 88:062702, 2013.

22. Gao-Jin Li and Arezoo M. Ardekani. Hydrodynamic interaction of microswimmers near a wall. Phys. Rev. E, 90:013010, 2014

23. Nima Sharifi-Mood, Ali Mozaffari, and Ubaldo M. CrdovaFigueroa. Pair interaction of catalytically active colloids: from assembly to escape. Journal of Fluid Mechanics, 798:910-954, 2016.

24. Dario Papavassiliou and Gareth P. Alexander. Exact solutions for hydrodynamic interactions of two squirming spheres. Journal of Fluid Mechanics, 813:618-646, 2017.

25. Peter J. Mucha, Shang-You Tee, David A. Weitz, Boris I. Shraiman, and Michael P. Brenner. A model for velocity fluctuations in sedimentation. Journal of Fluid Mechanics, 501:71-104, 022004.

26. John J. Molina, Yasuya Nakayama, and Ryoichi Yamamoto. Hydrodynamic interactions of self-propelled swimmers. Soft Matter, 9:4923-4936, 2013.
27. Andreas Zöttl and Holger Stark. Hydrodynamics determines collective motion and phase behavior of active colloids in quasi-two-dimensional confinement. Phys. Rev. Lett., 112:118101, 2014.

28. Ricard Matas-Navarro, Ramin Golestanian, Tanniemola B Liverpool, and Suzanne M. Fielding. Hydrodynamic suppression of phase separation in active suspensions. Phys. Rev. E, 90:032304, 2014.

29. J.-B. Delfau, J. Molina, and M. Sano. Collective behavior of strongly confined suspensions of squirmers. Eur. Phys. Lett., 114(2):24001, 2016.

30. Saverio E Spagnolie and Eric Lauga. Hydrodynamics of self-propulsion near a boundary: predictions and accuracy of far-field approximations. Journal of Fluid Mechanics, 700:105-147, 2012.

31. D. Saintillan and M. J. Shelley. Complex Fluids in Biological Systems, chapter Theory of active suspensions, pages 319-355. Springer, 2015.

32. Joakim Stenhammar, Cesare Nardini, Rupert W. Nash, Davide Marenduzzo, and Alexander Morozov. Role of correlations in the collective behavior of microswimmer suspensions. Phys. Rev. Lett., 119:028005, 2017.

33. Natsuhiko Yoshinaga and Tanniemola B. Liverpool. Hydrodynamic interactions in dense active suspensions: From polar order to dynamical clusters. Phys. Rev. E, 96:020603(R), 2017.

34. Francisco Alarcon, Chantal Valeriani, and Ignacio Pagonabarraga. Morphology of clusters of attractive dry and wet self-propelled spherical particle suspensions. Soft Matter, 13:814-826, 2017.

35. E. L. Hill. The theory of vector spherical harmonics. Am. J. Phys., 22(4):211-214, 1954.

36. AR Edmonds. Angular momentum in quantum mechanics. Princeton Univ. Press, 1957.

37. J.R. Blake and A.T. Chwang. Fundamental singularities of viscous flow. Journal of Engineering Mathematics, 8(1):23-29, 1974.

38. DJ Jeffrey and Y Onishi. Calculation of the resistance and mobility functions for two unequal rigid spheres in low-reynolds-number flow. Journal of Fluid Mechanics, 139:261-290, 1984.

39. S. Kim and S.J. Karrila. Microhydrodynamics. Butterworth-Heinemann, New York, 1991.

40. OnShun Pak and Eric Lauga. Generalized squirming motion of a sphere. Journal of Engineering Mathematics, 88(1):1-28, 2014.

41. S. R. Majumdar. Slow motion of an incompressible viscous liquid generated by the rotation of two spheres in contact. Mathematika, 14:43-46, 61967.

42. M. E. O'Neill. On asymmetrical slow viscous flows caused by the motion of two equal spheres almost in contact. Mathematical Proceedings of the Cambridge Philosophical Society, 65:543-556, 31969.

43. MichaelE. O'Neill and SamirR. Majumdar. Asymmetrical slow viscous fluid motions caused by the translation or rotation of two spheres. part ii: Asymptotic forms of the solutions when the minimum clearance between the spheres approaches zero. Zeitschrift fr angewandte Mathematik und Physik ZAMP, 21(2):180-187, 1970.

44. MDA Cooley. The slow rotation in a viscous fluid of a sphere close to another fixed sphere about a diameter perpendicular to the line of centres. The Quarterly Journal of Mechanics and Applied Mathematics, 24(2):237-250, 1971. 
45. M. B. A. Cooley and M. E. O'Neill. On the slow motion of two spheres in contact along their line of centres through a viscous fluid. Mathematical Proceedings of the Cambridge Philosophical Society, 66:407-415, 91969.

46. D. J. Jeffrey. Low-reynolds-number flow between converging spheres. Mathematika, 29:58-66, 61982.

47. D.J. Jeffrey and Y. Onishi. The forces and couples acting on two nearly touching spheres in low-reynolds-number flow. Zeitschrift fr angewandte Mathematik und Physik ZAMP, 35(5):634-641, 1984.

48. James W. Swan, John F. Brady, Rachel S. Moore, and ChE 174. Modeling hydrodynamic self-propulsion with stokesian dynamics. or teaching stokesian dynamics to swim. Physics of Fluids, 23(7):071901, 2011.

49. M. E. O'Neill and K. Stewartson. On the slow motion of a sphere parallel to a nearby plane wall. J. Fluid Mech., 27:705-724, 31967.

50. M. D. A. Cooley and M. E. O'Neill. On the slow rotation of a sphere about a diameter parallel to a nearby plane wall. IMA Journal of Applied Mathematics, 4(2):163-173, 1968.

51. Alois Würger. Hydrodynamic boundary effects on thermophoresis of confined colloids. Phys. Rev. Lett., 116:138302, 2016.

52. JF Brady and G. Bossis. Stokesian dynamics. Ann. Rev. Fluid Mech., 20(1):111-157, 1988.

53. J. R. Melrose and R. C. Ball. The pathological behaviour of sheared hard spheres with hydrodynamic interactions. Europhysics Letters, 32(6):535, 1995.

54. E. Yariv and H. Brenner. Near-contact electrophoretic motion of a sphere parallel to a planar wall. J. Fluid Mech., 484:85-111, 62003.

55. Raymond G. Cox and Howard Brenner. The slow motion of a sphere through a viscous fluid towards a plane surfaceii small gap widths, including inertial effects. Chemical Engineering Science, 22(12):1753 - 1777, 1967.

56. M. D. A. Cooley and M. E. O'Neill. On the slow motion generated in a viscous fluid by the approach of a sphere to a plane wall or stationary sphere. Mathematika, 16:37-49, 61969.

57. Rajesh Singh and R. Adhikari. Generalized stokes laws for active colloids and their applications. arXiv:1603.05735 2016.

58. Kengo Ichiki. Improvement of the stokesian dynamics method for systems with a finite number of particles. Journal of Fluid Mechanics, 452:231-262, 2002.

59. P. Mazur and W. van Saarloos. Many-sphere hydrodynamic interactions and mobilities in a suspension. Physica A: Statistical Mechanics and its Applications, 115(1-2):2157,1982

60. Anthony J. C. Ladd. Hydrodynamic transport coefficients of random dispersions of hard spheres. The Journal of Chemical Physics, 93(5):3484-3494, 1990.

61. F. Alarcón and I. Pagonabarraga. Spontaneous aggregation and global polar ordering in squirmer suspensions. Journal of Molecular Liquids, 185:56 - 61, 2013.

62. Fernando Peruani, Andreas Deutsch, and Markus Bär. Nonequilibrium clustering of self-propelled rods. Phys. Rev. E, 74(3):030904, 2006.

63. Johannes Blaschke, Maurice Maurer, Karthik Menon, Andreas Zöttl, and Holger Stark. Phase separation and coexistence of hydrodynamically interacting microswimmers. Soft Matter, 12:9821-9831, 2016.
64. P G Saffman and M Delbrck. Brownian motion in biological membranes. Proc. Nat. Acad. Sci., 72(8):3111-3113, 1975.

65. G. W. Gardiner. Handbook of Stochastic Methods: for Physics, Chemistry and the Natural Sciences. Springer, New York, 1985.

66. Arthur A. Evans, Takuji Ishikawa, Takami Yamaguchi, and Eric Lauga. Orientational order in concentrated suspensions of spherical microswimmers. Physics of Fluids, 23(11):111702, 2011.

67. Norihiro Oyama, John Jairo Molina, and Ryoichi Yamamoto. A binary collision route for purely hydrodynamic orientational ordering of microswimmers. arXiv:1606.03839, 2016.

68. R.C. Ball and J.R. Melrose. A simulation technique for many spheres in quasi-static motion under frame-invariant pair drag and brownian forces. Physica A, 247(1-4):444472, 1997.

69. H. Chaté, F. Ginelli, G. Grégoire, F. Peruani, and F. Raynaud. Modeling collective motion: variations on the vicsek model. The European Physical Journal B, 64(3-4):451-456, 2008.

70. Joakim Stenhammar, Davide Marenduzzo, Rosalind J. Allen, and Michael E. Cates. Phase behaviour of active brownian particles: the role of dimensionality. Soft Matter, 10:1489-1499, 2014.

71. Marco Leoni and Tanniemola B. Liverpool. Swimmers in thin films: From swarming to hydrodynamic instabilities. Phys. Rev. Lett., 105:238102, Dec 2010.

72. R. Aditi Simha and Sriram Ramaswamy. Hydrodynamic fluctuations and instabilities in ordered suspensions of selfpropelled particles. Phys. Rev. Lett., 89(5):058101, 2002.

73. Broto Tjipto-Margo, Glenn T Evans, Michael P Allen, and Daan Frenkel. Elastic constants of hard and soft nematic liquid crystals. The Journal of Physical Chemistry, 96(10):3942-3948, 1992.

74. R Voituriez, JF Joanny, and J Prost. Spontaneous flow transition in active polar gels. EPL (Europhysics Letters), 70(3):404, 2005.

75. A. C. Maggs and V. Rossetto. Local simulation algorithms for coulomb interactions. Phys. Rev. Lett., 88:196402, Apr 2002.

76. Natsuhiko Yoshinaga. Spontaneous motion and deformation of a self-propelled droplet. Phys. Rev. E, 89:012913, 2014.

77. Margaret Stimson and GB Jeffery. The motion of two spheres in a viscous fluid. Proceedings of the Royal Society of London. Series A, 111(757):110-116, 1926.

78. A.J. Goldman, R.G. Cox, and H. Brenner. Slow viscous motion of a sphere parallel to a plane wall - i motion through a quiescent fluid. Chemical Engineering Science, 22(4):637 - 651, 1967.

79. G. B. Jeffery. The rotation of two circular cylinders in a viscous fluid. Proceedings of the Royal Society of London A: Mathematical, Physical and Engineering Sciences, 101(709):169-174, 1922.

80. D. J. Jeffrey and Y. Onishi. The slow motion of a cylinder next to a plane wall. The Quarterly Journal of Mechanics and Applied Mathematics, 34(2):129-137, 1981.

81. J. Yang, C. W. Wolgemuth, and G. Huber. Force and torque on a cylinder rotating in a narrow gap at low reynolds number: Scaling and lubrication analyses. Physics of Fluids, 25(5):051901, 2013. 
82. R. Cardinaels and H. A. Stone. Lubrication analysis of interacting rigid cylindrical particles in confined shear flow. Physics of Fluids, 27(7):072001, 2015. 